\title{
Infrared behavior of Weyl gravity: Functional renormalization group approach
}

\author{
Petr Jizba@, ${ }^{*}$ Lesław Rachwał®, ${ }^{\dagger}$ and Jaroslav Kňap ${ }^{\ddagger}$ \\ FNSPE, Czech Technical University in Prague, Břehová 7, 11519 Praha 1, Czech Republic
}

(Received 23 December 2019; accepted 27 January 2020; published 24 February 2020)

\begin{abstract}
Starting from an ultraviolet fixed point, we study the infrared behavior of quantum Weyl gravity in terms of a functional renormalization group (RG) flow equation. To do so, we employ two classes of Bach-flat backgrounds, namely maximally symmetric spacetimes and Ricci-flat backgrounds in the improved one-loop scheme. We show that in the absence of matter fields and with a topological term included, the effective action exhibits dynamical breaking of scale symmetry. In particular, it is shown that apart from a genuine IR fixed point that is reached at a zero value of the running scale, the $\mathrm{RG}$ flow also exhibits bouncing behavior in the IR regime. We demonstrate that both $\beta_{C}$ and $\beta_{E}$ reach the RG turning point (almost) simultaneously at the same finite energy scale, irrespective of the chosen background. The IR fixed point itself is found to be IR stable in the space of the considered couplings. Ensuing scaling dimensions of both operators are also computed. Salient issues, including the connection of the observed bouncing RG flow behavior with holography and prospective implications in early Universe cosmology, are also briefly discussed.
\end{abstract}

DOI: 10.1103/PhysRevD.101.044050

\section{INTRODUCTION}

The current constraints from Planck measurements of the $\mathrm{CMB}$ anisotropies indicate that the cosmological perturbations are (nearly) scale invariant with the value of the scalar spectral index $n_{s}=0.965 \pm 0.004$ (with a $68 \%$ confidence level) [1]. This is tantalizingly close to $n_{s}=1$, which corresponds to the exact scale-invariant fluctuations. This fact suggests that it might be useful to describe the very early stage of the Universe in terms of some (possibly even effective) scale-invariant gravitational theories.

Theories with (classical or quantum) scale invariance have a long and venerable history. Apart from the fact that they are instrumental in providing a dynamical origin of mass scales [2-8], they have a number of further desirable features; for instance, they provide an appealing framework for addressing the hierarchy problem [9], lead to naturally flat inflationary potentials [10], furnish dark matter candidates $[11,12]$ or even provide a viable alternative for dark matter itself [13-15].

In exactly scale-invariant theories, no energy scale is preferred since all are treated on equal footing. So, in order

\footnotetext{
p.j.jizba@fjfi.cvut.cz grzerach@gmail.com

*knapjaro@fjfi.cvut.cz
}

Published by the American Physical Society under the terms of the Creative Commons Attribution 4.0 International license. Further distribution of this work must maintain attribution to the author(s) and the published article's title, journal citation, and DOI. Funded by SCOAP . to describe the appearance of physical energy scales (as observed at low enough energies), any phenomenologically viable scale-invariant quantum gravity theory has to exhibit the scale symmetry breaking in one way or another. For instance, this symmetry can be explicitly broken by mass terms or via dimensional transmutation. The latter could happen either perturbatively through a Coleman-Weinberg mechanism or nonperturbatively [as it happens, for instance, by monopole condensation in quantum chromodynamics (QCD)]. In these cases, the scale symmetry is typically quantum anomalous (by virtue of trace anomaly), and it is manifest only in the vicinity of nontrivial fixed points [16] of the renormalization group (RG) flow. Only in exceptional circumstances, such as a $\mathcal{N}=4$ supersymmetric Yang-Mills theory, one has an exact cancellation of this anomaly in the quantum field theory (QFT) framework. It should perhaps be stressed that the trace anomaly is not a precursor of the spontaneous symmetry breaking (SSB) of scale invariance; i.e., the situation where a vacuum expectation value (VEV) of some operator (order-parameter operator) supplies the needed dimensionful parameter. In fact, the symmetry that is broken in SSB is a global symmetry, not the local one (Elitzur's theorem [17]), and the trace anomaly is generally considered as harmless for global scale invariance [18]. For instance, for quantum Weyl gravity (QWG) (or quantum conformal gravity), a typical imprint of the trace anomaly in the scale-symmetric phase of QWG is the appearance of the $R^{2}$ (and GaussBonnet $E$ ) term in the renormalized action, even if this term is not implied by the local Weyl symmetry. Actually, the $R^{2}$ local term is globally scale invariant in spacetime with 
$d=4$ dimensions, but not locally, while the variation of the Gauss-Bonnet term in $d=4$ vanishes, so this term is invariant with respect to any symmetry transformation (both global and local). In addition, when the scale invariance is spontaneously broken then the (conformal) Ward identities imply that the trace anomalies in the symmetric and broken phases are matched, though the analytical structure of the correlators is different in the two respective phases [19].

The inclusion of gravity in the scale-invariant framework offers far-reaching consequences. On the one hand, the breaking of the scale symmetry translates into the appearance of a pseudo-Goldstone boson (dilaton) which, due to its small mass, could potentially contribute to the earlyand late-time acceleration of the Universe or to counting of the number of relativistic degrees of freedom at big bang nucleosynthesis and recombination. On the other hand, the Standard Model of particle physics faces the problem of stability of the Higgs mass against radiative corrections (the fine-tuning problem). If the full quantum theory, including gravity, is scale invariant, and the scale symmetry is spontaneously broken, then the Standard-Model Higgs mass is protected from radiative corrections by an exact dilatational symmetry [20], cf. also [21].

Our modus operandi here will be based on the assumption that QWG can serve as a pertinent scaleinvariant gravitational theory at high (preinflationary) energies. We will follow this premise, to address the ensuing low-energy phenomenology. Let us first note that since the scale invariance in the QWG is gauged (i.e., appears in the local version), the Weyl gravity is a very special theory and, in fact, unique among all higher derivative gravity (HDG) theories. This theory possesses even bigger symmetry, namely conformal invariance, which naturally appears in the local form. That is why some authors [16] prefer to call this theory as conformal gravity. Actually, in $d=4$, all HDG theories with four derivatives of the metric field are invariant under rigid scale transformations. In a sense, the Weyl gravity is the simplest among HDG theories as it is determined in $d=4$ by a single (inevitably) dimensionless coupling parameter. This should be compared, e.g., with two couplings present in a generic Stelle-type four-derivative theory [22]. It should also be stressed that the Weyl gravity has a different counting of dynamical degrees of freedom ${ }^{1}$ that cannot be obtained by any limiting procedure from a generic HDG theory (e.g., by taking couplings in front of gauge-symmetry breaking terms to zero) due to the van Dam-Veltman-Zakharov discontinuity [23] (see also [24] and references therein).

\footnotetext{
${ }^{1}$ For example, in $d=4$ there are 6 degrees of freedom in contrast to 8 , which are typical for other HD gravitational theories with four derivatives.
}

At the quantum level, Weyl gravity shares the same fate with all other higher derivative (HD) theories, namely the unitarity is in danger because there are perturbative states with negative kinetic energy (ghost states [22]). As a matter of fact, QWG has 6 perturbative degrees of freedom from which 2 are ghost degrees of freedom corresponding to the spin-2 massless particle. The conventional optical theorem, in turn, implies that the $S$ matrix is in such cases nonunitary. Obviously, ghosts are undesirable and various approaches have been invoked to remove them (or their effects) from the observable predictions of the theory. Diverse cures have been proposed in the literature for dealing with the ghosts issue: the Lee-Wick prescription [25], fakeons [26], the disappearance of unstable fluctuations in nontrivial backgrounds [27], nonperturbative numerical methods [28-31], benign ghosts [32-34], nonlocal gravity [35,36], non-Hermitian quantum gravity [13-15], etc. (see also $[37,38]$ and citations therein). One might even entertain the idea that unitarity in quantum gravity is not a fundamental concept [39-41]. So far, none of the proposed solutions conclusively solves the problem.

It is quite possible that the unitarity problem might eventually be not as harmful as it seems. One appealing possibility for the resolution of the unitarity issue is Weinberg's asymptotic safety (AS) scenario [42]. The AS proposal/conjecture is based on the idea that quantum gravity develops a nontrivial fixed point (FP) of the RG in the ultraviolet (UV) regime. Since the conjectured FP is non-Gaussian, then the couplings attain finite (possibly nonsmall) values at the end of the RG flow. Consequently, this proposal requires a nonperturbative analysis of the RG flow. Fortunately, an appropriate tool for this task-the so-called functional (or exact) renormalization group (FRG), has become available recently $[43,44]$. Though technically still involved, there is a strong hope that when the nontrivial FP is found then the corresponding gravity theory will behave in a controllable way, and the FP will determine fully the nonperturbative spectrum, solve the problem with unitarity, and tame the divergences since the RG flow stops at the FP $[45,46]$. This type of scenario (reinforced by a condition of a finite dimensionality of the critical surface on which assumed FP lies) is well-known from the QFT description of critical phenomena in condensed matter, where the non-Gaussian FP provides a welldefined theory in the UV (or IR) regime [47]. Moreover, when the theory sits at the FP, then its symmetry is often enhanced and ensuing FP's are described by quantum scale invariant or even conformal field theories (CFT). This is one characteristic way how the exact conformal symmetry may show up on the quantum level.

Instead of debating various attitudes that can be taken toward the ghost-unitarity issue, our aim is more modest. We wish to explore, via FRG the low-energy phenomenology of the QWG and see whether it can provide a 
realistic cosmology and what role (if any) is played by ghost fields. The aim of this paper (first in a series of two) is twofold:

(a) Let us assume that we start from the UV FP where the would-be quantum gravity has an exact scale invariance. In order not to invoke any unwarranted structure, we consider only purely metric-field-based gravity without any matter field. The UV FP in question might be, for instance, one of the critical points in a series of hypothetical phase transitions that the Universe has undergone in the very early (preinflationary) stage of its evolution. Out of many scale-invariant HDG candidate theories, we choose to work with the simplest one, namely the one that has only one coupling constant. The latter corresponds to the quantum Weyl gravity. Precisely at the fixed point, the QWG has exact local scale invariance. If the UV fixed point is non-Gaussian (we shall see it is not), it should be of a Banks-Zaks type [48] — so that the perturbative analysis would still be applicable. Existence of such UV fixed point for QWG is only conjectured here but there are various indications that this might be, indeed, the case $[49,50]$. We start with this working hypothesis and let the theory flow toward infrared (IR) energy scales. In the close vicinity of the UV FP, the Weyl symmetry in the renormalized Lagrangian is still preserved as only the Gauss-Bonnet term is generated in the process. Corrections explicitly violating local scale symmetry, like the $R^{2}$ term, appear only at the second or higher loop level [16]. This is a consequence of the fact that local scale invariance is preserved at the one-loop level, while local scale symmetry violating corrections are inevitably expected (though at higher-loop levels) due to a nonvanishing trace anomaly at the one-loop level. In this close neighborhood of the UV FP, we choose a truncation ansatz for the effective action that will be used to set up the FRG flow equation. In passing, we stress that we analyze RG flow in the QWG which has only dimensionless couplings; hence, most of the objections raised in the literature (cf., e.g., [51]) against the asymptotic safety program do not apply here.

(b) In the next step, we will solve the ensuing RG flow equation algebraically for the two $\beta$ functions involved and show that there exists a non-Gaussian IR fixed point where both $\beta$ functions simultaneously disappear. This IR FP represents a critical point after which the (global) scale invariance is broken. The fact that the scale invariance gets broken is reflected via appearance of the related (composite) order-parameter field of the HubbardStratonovich (HS) type which mediates a dynamical breakdown of the scale symmetry. In the broken phase, the order-parameter field acquires a nontrivial vacuum expectation value (VEV) via dimensional transmutation. This might in turn provide a key scalar degree of freedom needed, for instance, in various cosmologically feasible inflationary scenarios. The latter point will be explored in more detail in a subsequent paper.

The structure of the paper is as follows: to set the stage, we discuss in the next section some fundamentals of the QWG that will be needed in sections to follow. In particular, we outline the route to quantization of Weyl gravity (WG) via functional integrals and stress some of potential problems encountered en route. We also emphasize a subtle fact that a nondynamical spurion scalar field can be introduced in the QWG via the HS transformation without spoiling the particle spectrum, (presumed nonperturbative) unitarity, and perturbative renormalizability. The HS field is actually an imprint of a scalar degree of freedom that would normally appear in the theory should the Weyl symmetry not decouple it from the on shell spectrum. A second part of Sec. II is dedicated to the York field decomposition in Weyl gravity. In Sec. III A, we employ this decomposition to construct the one-loop partition functions for the maximally symmetric spaces (MSS) and Ricci-flat backgrounds. We proceed in Sec. III B by constructing the FRG flow equation (in an Euclidean setting) for the QWG. To this end, we use the truncation prescription implied by the one-loop effective action. We further enhance this by including two nonperturbative effects, namely threshold phenomena and the effect of anomalous dimension of graviton field. In Sec. IV, we analyze the $\beta$ functions $\beta_{C}$ and $\beta_{E}$ that are affiliated with the Weyl tensor square and Gauss-Bonnet terms, respectively. In particular, we show that apart from a genuine IR fixed point that is reached at zero value of the running scale, the RG flow also exhibits bouncing behavior in the vicinity of the IR FP. We demonstrate that both $\beta_{C}$ and $\beta_{E}$ reach the RG bounce fixed point (almost) simultaneously at the same finite energy scale, irrespectively of the chosen background. The IR fixed point itself is shown to be IR stable. Ensuing scaling dimensions for the two operators are also computed. Finally, Sec. V summarizes our results and discusses prospective implications for early Universe cosmology. For the reader's convenience, the paper is accompanied with Supplemental Material (SM) [52] that clarifies some technical and conceptual details needed in the main text.

\section{QUANTUM WEYL GRAVITY}

\section{A. Classical Weyl gravity}

The WG is a pure metric theory that is invariant not only under the action of the diffeomorphism group, but also under Weyl rescaling of the metric tensor by the local smooth functions $\Omega(x): g_{\mu \nu}(x) \rightarrow \Omega^{2}(x) g_{\mu \nu}(x)$. The simplest WG action functional in four spacetime dimensions that is both diffeomorphism and Weyl-invariant has the form $[64,65]$, 


$$
S=-\frac{1}{4 \alpha^{2}} \int d^{4} x \sqrt{|g|} C_{\mu \nu \rho \sigma} C^{\mu \nu \rho \sigma}
$$

where $C_{\mu \nu \rho \sigma}$ is the Weyl tensor which can be written as

$C_{\mu \nu \rho \sigma}=R_{\mu \nu \rho \sigma}-\left(g_{\mu[\rho} R_{\sigma] \nu}-g_{\nu[\rho} R_{\sigma] \mu}\right)+\frac{1}{3} R g_{\mu[\rho} g_{\sigma] \nu}$,

with $R_{\mu \nu \rho \sigma}$ being the Riemann curvature tensor, $R_{\mu \rho}=$ $R_{\mu \nu \rho}{ }^{\nu}$ the Ricci tensor, and $R=R_{\mu}{ }^{\mu}$ the scalar curvature. Here and throughout the text, we use the timelike metric signature $(+,-,-,-)$ whenever pseudo-Riemannian (Lorentzian) manifolds are considered. The dimensionless coupling constant $\alpha$ is conventionally chosen so as to mimic the Yang-Mills action. On the other hand, in order to make a connection with the usual RG methodology, it will be more convenient to consider the inverse of the coupling $\alpha^{2}$. We will denote the coupling in front of the Weyl square term in Eq. (1) as $\omega_{C}$ via the identification $\omega_{C} \equiv 1 /\left(4 \alpha^{2}\right)$.

As for the notation for various scalar invariants (with four derivatives of the metric tensor), we accept the following conventions: for the square of the Riemann tensor contracted naturally (preserved order of indices), that is $R_{\mu \nu \rho \sigma} R^{\mu \nu \rho \sigma}$, we use the symbol $R_{\mu \nu \rho \sigma}^{2}$; the square of the Ricci tensor $R_{\mu \nu} R^{\mu \nu}$, we denote by simply $R_{\mu \nu}^{2}$; the square of the Ricci curvature scalar is always $R^{2}$, while for the Weyl tensor square (with a natural contraction of indices) $C_{\mu \nu \rho \sigma} C^{\mu \nu \rho \sigma}$, we employ a shorthand and schematic notation $C^{2}$. When the latter is treated as a local invariant (not under a volume integral, so without the possibility of integrating by parts) in $d=4$ dimensions, one finds the following expansion of the $C^{2}$ invariant into standard invariants quadratic in curvature:

$$
C^{2}=R_{\mu \nu \rho \sigma}^{2}-2 R_{\mu \nu}^{2}+\frac{1}{3} R^{2}
$$

Finally, we will also need yet another important combination of the quadratic curvature invariants, namely,

$$
E=R_{\mu \nu \rho \sigma}^{2}-4 R_{\mu \nu}^{2}+R^{2},
$$

which in $d=4$ is the integrand of the Euler (or GaussBonnet) invariant [49],

$$
\chi=\frac{1}{32 \pi^{2}} \int d^{4} x \sqrt{|g|} E .
$$

In the following, we will call the invariant $E$ in the action as a Gauss-Bonnet term. With the help of the Chern-GaussBonnet theorem, one can cast the Weyl action $S$ into an equivalent form (modulo topological term),

$$
S=-\frac{1}{2 \alpha^{2}} \int d^{4} x \sqrt{|g|}\left(R_{\mu \nu}^{2}-\frac{1}{3} R^{2}\right) .
$$

It should be stressed that both (1) and (6) are Weyl invariant only in $d=4$ dimensions. In fact, under the (conformal or Weyl) transformation $g_{\mu \nu} \rightarrow \Omega^{2} g_{\mu \nu}$, the densitized square of the Weyl tensor transforms as

$$
\sqrt{|g|} C^{2} \rightarrow \Omega^{d-4} \sqrt{|g|} C^{2}
$$

in a general dimension $d$ of spacetime, while $\sqrt{|g|} E$ supplies topological invariant only in $d=4$ (the variation of this last term is a total derivative). This is particularly important to bear in mind during the quantization where (similarly as in the Yang-Mills theories), one should choose such a regularization method that preserves the local gauge symmetry of the underlying Lagrangian and thereby does not introduce any unwarranted symmetry breaking terms. For this reason, one should preferentially rely on fixed-dimension renormalization schemes (as done throughout this paper) and avoid, e.g., dimensional regularization.

A variation of $S$ with respect to the metric yields the field equation (Bach vacuum equation),

$$
2 \nabla_{\kappa} \nabla_{\lambda} C^{\mu \kappa \nu \lambda}-C^{\mu \kappa \nu \lambda} R_{\kappa \lambda} \equiv B^{\mu \nu}=0,
$$

where $B^{\mu \nu}$ is the Bach tensor and $\nabla_{\alpha}$ is the usual covariant derivative (with a Levi-Civita connection). We remind that this form of the equation of motion (EOM) is specific only to four spacetime dimensions. Moreover, the Bach tensor is always traceless $\left(B^{\mu}{ }_{\mu}=0\right)$ as a consequence of conformal symmetry and also is divergence-free $\left(\nabla_{\mu} B^{\mu \nu}=0\right)$ as a consequence of diffeomorphism symmetry. One can also show that in $d=4$, one has $B^{\mu \nu}=B^{\nu \mu}$ as a consequence of being a variational derivative of the action $S$ with respect to a symmetric metric tensor $g_{\mu \nu}$. When on a given background $B^{\mu \nu}=0$ (i.e., this configuration is a classical vacuum solution in Weyl gravity), then we say that it is Bach flat.

\section{B. Quantization of Weyl gravity}

We formally define a quantum field theory of gravity by a functional integral $(\hbar=c=1)$,

$$
Z=\sum_{i} \int_{\Sigma_{i}} \mathcal{D} g_{\mu \nu} e^{i S}
$$

Here, $\mathcal{D} g_{\mu \nu}$ denotes the functional-integral measure whose proper treatment involves the Faddeev-Popov gauge fixing of the gauge symmetry diff $\times \operatorname{Weyl}\left(\Sigma_{i}\right)$ plus the ensuing Faddeev-Popov determinant [16]. As for the local factors $\left[-\operatorname{det} g_{\mu \nu}(x)\right]^{\omega}$ in the measure, we choose to work with the 
De Witt convention [66]: $\omega=(d-4)(d+1) / 8$. In this case, the local factor does not contribute when the fixeddimension renormalization in $d=4$ is employed.

The sum in (9) is a sum over four topologies, that is, the sum over topologically distinct manifolds $\Sigma_{i}$ (analogue to the sum over genera in string theory or the sum over homotopically inequivalent vacua in the Yang-Mills theory), which can potentially contain topological phase factors, e.g., the Euler-Poincaré characteristic of $\Sigma_{i}$, cf. Refs. [67]. ${ }^{2}$

For future convenience, we note that the $R^{2}$ part in the Weyl action $S$ in (6) can be further decomposed with the help of the Hubbard-Stratonovich (HS) transformation $[10,69-71]$ as

$$
\begin{aligned}
\exp \left(i S_{R}\right) & \equiv \exp \left(\frac{i}{6 \alpha^{2}} \int d^{4} x \sqrt{|g|} R^{2}\right) \\
& =\int \mathcal{D} \phi \exp \left[-i \int d^{4} x \sqrt{|g|}\left(\phi R+\frac{3}{8 \omega_{C}} \phi^{2}\right)\right] .
\end{aligned}
$$

It is not difficult to see that the essence of the HS transformation (10) is a straightforward manipulation of a functional Gaussian integral (shifting the quadratic trinomial in the exponent). Although an auxiliary HS field $\phi(x)$ does not have a bare kinetic term, one might expect that due to radiative corrections it will develop in the IR regime a gradient term which will then allow us to identify the HS boson with a genuine propagating mode. This scenario is, in fact, well-known from the condensed matter theory. A quintessential example of this is obtained when the BCS superconductivity is reduced to its low-energy effective level. There, the HS boson coincides with the disordered field whose dynamics is described via the celebrated Ginzburg-Landau equation [71,72].

The $\phi$ field can be separated into a background field $\langle\phi\rangle$ corresponding to a VEV of $\phi$ plus fluctuations $\delta \phi$. Since $\langle\phi\rangle$ is dimensionful, it must be zero in the case when the theory is scale invariant. On the other hand, when the scale invariance is broken, $\phi$ will develop a nonzero VEV. So, the HS field $\phi$ plays the role of the order-parameter field. The inner workings of this mechanism were illustrated in Ref. [10], where it was shown than on the flat background $\phi$ develops (in the broken phase) a nonzero VEV. With the

\footnotetext{
${ }^{2}$ The sum over four topologies is a problematic concept since four manifolds are generally unclassifiable - that is, there is no algorithm that can determine whether two arbitrary four manifolds are homeomorphic. On the other hand, simply connected compact topological four manifolds are classifiable in terms of Casson handles shown by M. H. Freedman [68], which can be applied in functional integrals in Euclidean gravity. In the Lorentzian case, one simply restricts oneself to some subset of four manifolds. If this subset is closed under a composition of the functional integral, then a theory thereby obtained is at least naively self-consistent.
}

benefit of hindsight, we further introduce an arbitrary "mixing" hyperbolic angle $\vartheta \in(-\infty, \infty)$ and write formally

$$
S_{R}=S_{R} \cosh ^{2} \vartheta-S_{R} \sinh ^{2} \vartheta
$$

Applying now the HS transformation to the $S_{\mathrm{R}} \sinh ^{2} \vartheta$ part, we get

$$
\begin{aligned}
S_{R}= & -\int d^{4} x \sqrt{|g|} \phi R+\frac{2 \omega_{C} \cosh ^{2} \vartheta}{3} \int d^{4} x \sqrt{|g|} R^{2} \\
& +\frac{3}{8 \omega_{C} \sinh ^{2} \vartheta} \int d^{4} x \sqrt{|g|} \phi^{2} .
\end{aligned}
$$

Although the full theory described by the action $S$ is independent of the mixing angle $\vartheta$, truncation of the perturbation series after a finite loop order in the fluctuating metric field will destroy this independence. The optimal result is reached by employing the principle of minimal sensitivity $[73,74]$ known from the RG calculus. There, if a perturbation theory depends on some unphysical parameter (as $\vartheta$ in our case), the best result is achieved if each order has the weakest possible dependence on the parameter $\vartheta$. Consequently, at each loop order, the value of $\vartheta$ is determined from the vanishing of the corresponding derivative of effective action.

As discussed in [10], the fluctuations of the metric $g_{\mu \nu}$ can make $\langle\phi\rangle$ not only nonzero but one can also find a set of parameters in a model's parameter space for which $\langle\phi\rangle \sim M_{\mathrm{P}}^{2}$ where $M_{\mathrm{P}}=2.44 \times 10^{18} \mathrm{GeV}$ is related to the Planck energy scale. Consequently, Newton's constant $\kappa_{N}$ is dynamically generated. Owing to the last term in (12), the existence of dynamical dark energy (a dynamical cosmological constant) is also an automatic consequence of the theory. In addition, by assuming that in the broken phase a cosmologically relevant metric is that of the FriedmannLemaitre-Robertson-Walker (FLRW) type, then, modulo a topological term, the additional constraint,

$$
\int d^{4} x \sqrt{|g|} 3 R_{\mu \nu}^{2}=\int d^{4} x \sqrt{|g|} R^{2}
$$

holds due to a conformal flatness of the FLRW metric $[75,76]$. It was argued in [10] that from (6) and (12) one obtains in the broken phase the effective gravitational action of the form,

$$
S=-\frac{1}{2 \kappa_{N}^{2}} \int d^{4} x \sqrt{|g|}\left(R-\xi^{2} R^{2}-2 \Lambda_{\mathrm{cc}}\right),
$$

where both $\kappa_{N}$ (Newton's constant) and $\xi$ (Starobinsky's parameter) are dynamically generated. Note that $\xi$ has the dimension of an inverse mass and by the Planck satellite data $\xi / \kappa_{N} \sim 10^{5}$ (cf. Ref. [1]). The action (14) is nothing but the Starobinsky action with the cosmological constant. We stress that the cosmological constant $\Lambda_{\mathrm{cc}}$ is entirely of 
a geometric origin (it descends from the QWG), and it enters with the opposite sign in comparison with the usual mattersector induced (i.e., de Sitter) cosmological constant.

In the following sections, we will analyze in more detail the FP corresponding to the spontaneous symmetry breakdown of scale invariance in QWG. To reinforce our conclusions, we will use two nontrivial classes of Bachflat backgrounds (i.e., solutions of classical Bach vacuum equation), namely MSS and Ricci-flat backgrounds. ${ }^{3}$ The ensuing cosmological implications that are related to the broken phase of QWG will be discussed in the successive paper.

\section{York decomposition}

To avoid issues related to the renormalization of nonphysical sectors [i.e., Faddeev-Popov (FP) ghosts and longitudinal components of the metric field], it will be convenient in our forthcoming reasonings to employ the York decomposition of the metric fluctuations $h_{\mu \nu}$ defined as

$$
g_{\mu \nu}=g_{\mu \nu}^{(0)}+h_{\mu \nu}
$$

where we have denoted the background metric as $g_{\mu \nu}^{(0)}$. The York decomposition is then implemented in two steps [49]. In the first step (in $d=4$ spacetime dimensions), one rewrites the metric fluctuations as

$$
h_{\mu \nu}=\bar{h}_{\mu \nu}+\frac{1}{4} g_{\mu \nu} h
$$

where $h$ is a trace part of $h_{\mu \nu}$ and $\bar{h}_{\mu \nu}$ is the corresponding traceless part. More specifically,

$$
\begin{aligned}
g^{(0) \mu \nu} \bar{h}_{\mu \nu} & =\bar{h}_{\mu}{ }^{\mu}=0, \\
h & =g^{(0) \mu \nu} h_{\mu \nu}=h_{\mu}{ }^{\mu} .
\end{aligned}
$$

In our subsequent derivations, it always will be implicit that the Lorentz indices are raised or lowered via a background metric, i.e., via $g^{(0) \mu \nu}$ or $g_{(0) \mu \nu}$, respectively. Also, all covariant derivatives $\nabla_{\mu}$ below will be understood as taken with respect to the background metric. By the symbol $\square$, we denote the covariant box operator defined as $\square \equiv \nabla^{\mu} \nabla_{\mu}$. This is the so-called Bochner Laplacian operator, it is defined with the covariant derivative $\nabla$ built on the basis of the Levi-Civita connection in metric theories. It is a standard two-derivative operator, and it arises naturally as a covariant generalization of the d'Alembertian operator in general relativity.

\footnotetext{
${ }^{3}$ In Sec. A of SM [52], we show that in $d=4$, all Einstein spaces (characterized by the condition that $R_{\mu \nu}=\Lambda g_{\mu \nu}$ with $\Lambda=$ const), including both Ricci-flat and MSS manifolds as subcases, constitute an important class of Bach-flat backgrounds.
}

In the second step, one decomposes the traceless part into the transverse, traceless tensor $\bar{h}_{\mu \nu}^{\perp}$ and to parts carrying the longitudinal (unphysical) degrees of freedom, namely,

$$
\begin{aligned}
\bar{h}_{\mu \nu}= & \bar{h}_{\mu \nu}^{\perp}+\nabla_{\mu} \eta_{\nu}^{\perp}+\nabla_{\nu} \eta_{\mu}^{\perp} \\
& +\nabla_{\mu} \nabla_{\nu} \sigma-\frac{1}{4} g_{\mu \nu} \square \sigma .
\end{aligned}
$$

These mixed-longitudinal (and traceless) parts are written in terms of an arbitrary transverse vector field $\eta_{\mu}^{\perp}$ and a scalar (trace) degree of freedom $\sigma$. The last fields must satisfy the usual conditions of transversality and tracelessness, i.e.,

$$
\nabla^{\mu} \bar{h}_{\mu \nu}^{\perp}=0, \quad \nabla^{\mu} \eta_{\mu}^{\perp}=0, \quad \bar{h}_{\mu}^{\perp \mu}=0 .
$$

The true physical propagating field in QWG is the transverse and traceless field $\bar{h}_{\mu \nu}^{\perp} \equiv h_{\mu \nu}^{T T}$. Indeed, from the second variation of the Weyl action expanded around a generic background, it can be seen that $\bar{h}_{\mu \nu}^{\perp}$ is the only field component that propagates on a quantum level. The vector field $\eta_{\mu}^{\perp}$ and two scalar fields $h$ and $\sigma$ completely drop out from the expansion due to diffeomorphism and conformal invariance, respectively [77]. This is true around any background but particular examples are given in formulas below [in Eqs. (22) and (27)]. In this way, we do not have to consider neither trace nor longitudinal degrees of freedom, nor FP ghosts in quantum dynamics of the theory.

In addition, in Sec. III, we show that when the proper change of the integration measure under the functional integral (9) is employed, the fixings of gauges for both the diffeomorphism and conformal symmetry are done and the ensuing Faddeev-Popov determinant is taken into account, one indeed obtains precisely 6 propagating degrees of freedom (around flat spacetime background) — as expected in the QWG. This counting can further be bolstered by performing canonical Hamiltonian analysis and by counting constraints and their character. The latter leads again to 6 degrees of freedom, but in this case, the counting holds in any spacetime background [77]. Furthermore, we will see that the inclusion of the effect of (perturbative) zero modes will not change this counting, though it will be key for getting the correct expression for the partition function of the theory. In particular, in order to make the expression for the partition function nonsingular and nonvanishing, zero modes must be handled with care.

\section{FRG FLOW EQUATION FOR QWG}

In order to make a comparison with existent works on the FRG in the gravity context, we will perform our subsequent computations in an Euclidean setting, and our analysis will be performed exclusively in $d=4$ Euclidean space dimensions. 
By performing a Wick rotation from Minkowski space to Euclidean space, the question of the resulting metric signature arises. When one does, in a standard way, only the change of the time coordinate $t \rightarrow-i t_{E}$ (where $t_{E}$ is the name of the first coordinate in the Euclidean characterization of space), the resulting signature of the metric of space, is completely negative, that is $(-,-,-,-)$. It seems natural to define the corresponding GR-covariant d'Alembert operator as $\square_{E}=-\nabla^{\mu} \nabla_{\mu}$, where the generalization to curved Euclidean space is done by using a Bochner Laplacian. However, in all formulas that follow, we find more it convenient to use the following definition in the Euclidean signature $\square=\nabla^{\mu} \nabla_{\mu}$. We also remark that this last operator $\square$, if analyzed on the flat space background, has a negative semidefinite spectrum. We will also use a definition of the covariant Euclidean box operator (covariant Laplacian) $\Delta=\square=-\square_{E}$, and this last operator in the Euclidean flat space case has a spectrum which is characterized by $-k^{2}$, the four-dimensional Euclidean negative square of a four-momentum vector $k_{\mu}$ of eigenmode. From now on, the signature of the metric in Euclidean space will be taken to be $(+,+,+,+)$.

The aim of this section is to explore both the infrared and the ultraviolet behavior of the QWG by solving the FRG flow equation $[43,44]$ for the effective average action $\Gamma_{k}$, which reads

$$
\partial_{t} \Gamma_{k}=\frac{1}{2} \operatorname{Tr}\left[\partial_{t} R_{k}\left(\Gamma^{(2)}+R_{k}\right)^{-1}\right] .
$$

The IR cutoff $R_{k}$ suppresses the contribution of modes with small eigenvalues of the covariant Laplacian (or some other suitable differential operator) $-\Delta \ll k^{2}$, while the factor $\partial_{t} R_{k}$ removes contributions from large eigenvalues of $-\Delta \gg k^{2}$. In this way, the loop integrals are both IR and UV finite [78]. The second variational derivative $\Gamma^{(2)}$ depends on the background metric $g_{\mu \nu}^{(0)}$, which is the argument of the running effective action $\Gamma_{k}$, while $k$ is the running energy (momentum) scale or the momentum of a mode in the Fourier space. Here, we also use that $\partial_{t}=k \partial_{k}$.

Ideally, Eq. (20) would require calculation of the full resummed and RG-invariant effective action. It is, however, difficult to proceed analytically in this way, so we content ourselves here with the conventional procedure, according to which one should employ some well motivated ansatz for the effective action. In particular, in order to evaluate the rhs of Eq. (20), we employ here the Euclidean effective action in the enhanced one-loop scheme. Namely, we will consider one-loop effective action in which also the effects of the anomalous dimension and threshold phenomena are included. Ensuing truncation will thus go beyond the usual polynomial ansatz. On the other hand, for the lhs of (20), we project the flow on the subspace of the three invariants containing precisely four derivatives of the metric [Eqs. (3), (4), and $R^{2}$ invariant]. The reason why we consider effective action on the rhs being different from the effective action on the lhs is dictated by technical convenience. Namely, the rhs acts as source for the RG flow, while the lhs contains the desired structure of the effective action that is appropriate for the extraction of the $\beta$ functions. For more details, see Sec. III B.

\section{A. One-loop partition functions for MSS and Ricci-flat backgrounds}

For technical convenience, we choose to work with two classes of backgrounds, namely maximally symmetric spaces (MSS) and Ricci-flat manifolds. As we shall see, these backgrounds will provide complementary information on $\beta$ functions of the theory that will suffice to determine respective $\beta$ functions algebraically. To find corresponding effective actions and $\beta$ functions, we first compute related one-loop partition functions.

We remark here that the spectrum of the covariant box operator on nontrivial backgrounds depends on the boundary conditions put on this operator. In the Euclidean setup, we typically assume that our backgrounds are compact manifolds without boundaries. This defines what operators and which boundary conditions we speak about below when we take functional traces of such operators. These boundary conditions correspond to the asymptotic flatness and fall-off conditions for the fluctuations, when the space is considered in the decompactification limit and when it is Wick rotated back to the Minkowskian signature.

In order to proceed with the partition function computations, it is important to set up first a notation regarding the functional determinants and ensuing functional traces. We denote the type of space in which these determinants (and related traces) are to be evaluated by the subscript just after the symbol "det", while the superscript will always denote the standard power. The corresponding "internal traces" in the matrix space of fluctuations [corresponding to the field-theoretical number of degrees of freedom (d.o.f.) in such subspaces] will be denoted by the symbol "tr". We stress that the functional traces (denoted by "Tr") as used, for example, in the FRG flow equation (20), are different because they contain also the integrations over the background spacetime. There are a few subspaces of fluctuations in which we would like to consider our determinants. They mainly depend on the spin of the fluctuations and whether they are transverse, traceless, or completely unconstrained fields. We have a description of various fields in subscripts:

(i) 0 - spin- 0 scalar field, with 1 d.o.f., that is $\operatorname{tr}_{0} \hat{\mathbb{I}}=1$.

(ii) $1 \perp \equiv 1 T$ — spin- 1 constrained vector field $v_{\mu}^{T} \equiv v_{\mu}^{\perp}$ to be transverse $\nabla^{\mu} v_{\mu}^{T}=0$, with 3 d.o.f., that is $\operatorname{tr}_{1 \perp} \hat{\mathbb{I}}=3$.

(iii) 1 -spin-1 unconstrained vector field $v_{\mu}$, with 4 d.o.f., that is $\operatorname{tr}_{1} \hat{\Pi}=4$. 
(iv) $2 \perp \equiv 2 T T$ - spin-2 fully constrained tensor rank-2 symmetric field $h_{\mu \nu}^{T T} \equiv \bar{h}_{\mu \nu}^{\perp}$, with conditions to be traceless and transverse: $h^{T T}{ }_{\mu}{ }^{\mu}=\nabla^{\mu} h_{\mu \nu}^{T T}=0$, with 5 d.o.f., that is $\operatorname{tr}_{2 \perp} \hat{\mathbb{I}}=5$.

(v) $2 T$ - spin-2 partially constrained tensor rank-2 symmetric field $h_{\mu \nu}^{T} \equiv \bar{h}_{\mu \nu}$, with a condition to be only traceless $h^{T}{ }_{\mu}^{\mu}=0$, with 9 d.o.f., that is $\operatorname{tr}_{2 T} \hat{\mathbb{\Pi}}=9$.

(vi) 2-spin-2 fully unconstrained tensor rank-2 symmetric field $h_{\mu \nu}$, with 10 d.o.f., that is $\operatorname{tr}_{2} \hat{\mathbb{I}}=10$.

The reader should, in particular, notice the differences in the usage of "T" superscript for spin-1 and spin-2 fields. The above counting of d.o.f.'s in each subspace was, of course, specific, to $d=4$ dimensions. From this moment on, we will omit the superscript and subscript (0) from the background metric tensors $g^{(0) \mu \nu}$ or $g_{(0) \mu \nu}$, respectively.

Being forearmed with the above notation, we can now discuss the one-loop partition functions of the QWG on both aforementioned classes of spacetimes.

a) Maximally symmetric spaces-are defined so that the Riemann curvature tensor is fully expressible through the metric tensor,

$$
R_{\mu \nu \rho \sigma}=\frac{\Lambda}{d-1}\left(g_{\mu \rho} g_{\nu \sigma}-g_{\mu \sigma} g_{\nu \rho}\right),
$$

where $\Lambda$ is a (real) constant parameter. This implies that MSS are spaces of constant curvature. They are moreover conformally flat, i.e., $C_{\mu \nu \rho \sigma}=0$; hence, $C^{2}=0$. The examples of such spaces in the Euclidean setting are spheres and hyperboloids, while in the Minkowskian case, we can speak of de Sitter and anti-de Sitter spacetimes.

The second variation around the MSS background of QWG in $d=4$ in terms of $h_{\mu \nu}^{T T}$ fluctuations can be written in the form,

$$
\delta^{2} S=\int d^{4} x \sqrt{g} h^{T T \mu \nu}\left(\hat{\square}-\frac{2}{3} \Lambda \hat{\mathbb{I}}\right)\left(\hat{\square}-\frac{4}{3} \Lambda \hat{\mathbb{I}}\right) h_{\mu \nu}^{T T} .
$$

We write a hat over all differential operators (like $\nabla$ and $\square$ ) from here on to emphasize that they act in a suitable matrix space of fluctuations. After taking into account the Jacobian for a change of variables: $h_{\mu \nu} \mapsto\left\{h_{\mu \nu}^{T T}, \eta_{\mu}^{\perp}, h, \sigma\right\}$, together with the Faddeev-Popov (FP) determinant, and gauge fixings (for Weyl and diffeomorphic invariance), the functional integration gives the "one-loop partition function,"

$$
\tilde{Z}_{1-\text { loop }}^{2}=\frac{\operatorname{det}_{1 T}(\hat{\square}+\Lambda \hat{\mathbb{I}}) \operatorname{det}_{0}\left(\hat{\square}+\frac{4}{3} \Lambda \hat{\mathbb{I}}\right)}{\operatorname{det}_{2 T T}\left(\hat{\square}-\frac{2}{3} \Lambda \hat{\mathbb{I}}\right) \operatorname{det}_{2 T T}\left(\hat{\square}-\frac{4}{3} \Lambda \hat{\mathbb{I}}\right)} .
$$

This result is not yet correct as it does include a contribution from zero modes. Since the zero modes in the determinants render the partition function ill-defined (either singular or vanishing), they have to be excluded. In Sec. B of SM [52], we show that when the zero modes are properly accounted for, Eq. (23) changes into

$$
\begin{aligned}
Z_{1-\text { loop }}^{2}= & \frac{\operatorname{det}_{1}^{2}(\hat{\square}+\Lambda \hat{\mathbb{I}}) \operatorname{det}_{1}\left(\hat{\square}+\frac{1}{3} \Lambda \hat{\mathbb{I}}\right)}{\operatorname{det}_{2 T}\left(\hat{\square}-\frac{2}{3} \Lambda \hat{\mathbb{I}}\right) \operatorname{det}_{2 T}\left(\hat{\square}-\frac{4}{3} \Lambda \hat{\mathbb{I}}\right)} \\
& \times \frac{\operatorname{det}_{0}\left(\hat{\square}+\frac{4}{3} \Lambda \hat{\mathbb{I}}\right)}{\operatorname{det}_{0}(\hat{\square}+2 \Lambda \hat{\mathbb{I}})} .
\end{aligned}
$$

At this stage, it should be noted that both (23) and (24) imply 6 propagating degrees of freedom. This can be seen on the level of flat space (obtained simply by setting $\Lambda=0$ ) as well as on any MSS background. For this, it is enough to take the logarithm in the formula (24) of both sides and use properties of small internal traces in each subspace, and finally, exploit the formula for the number of degrees of freedom [16],

$$
N_{\text {d.o.f. }}=-\frac{\log Z^{2}}{\operatorname{Tr} \log \square},
$$

and mathematical identities,

$$
\begin{aligned}
& \log \operatorname{det}_{X} \hat{\square}=\operatorname{Tr}_{X} \log \hat{\square}, \\
& \operatorname{Tr}_{X} \log \hat{\square}=\operatorname{tr}_{X} \hat{\Pi} \cdot \operatorname{Tr} \log \square,
\end{aligned}
$$

valid on the flat spacetime for any subspace $X \in\{0,1 T$, $1,2 T T, 2 T, 2\}$.

b) Ricci-flat manifolds - are defined so that $R_{\mu \nu}=0$. The second variation around a Ricci-flat background in terms of $h_{\mu \nu}^{T T}$ fluctuations reads

$$
\delta^{2} S=\int d^{4} x \sqrt{g} h^{T T \mu \nu}(\hat{\square}-2 \hat{C})^{2} h_{\mu \nu}^{T T}
$$

Analogously as in the previous MSS spacetimes, we can now employ the Jacobian of the transformation to the variables used in the York decomposition, together with the FP determinant and fixing of local symmetries to obtain

$$
Z_{1-\text { loop }}^{2}=\frac{\operatorname{det}_{1}^{3} \hat{\square} \operatorname{det}_{0}^{2} \hat{\square}}{\operatorname{det}_{2}^{2}(\hat{\square}-2 \hat{C})} .
$$

In these spacetimes, there is no correcting contribution from zero modes. Operator $(\hat{\square}-2 \hat{C})$ in Eq. (27) represents the Laplacian and the matrix of the Weyl tensor on the background vector bundle acting on tensor fields such as $h_{\mu \nu}^{T T}$, which are transverse and traceless. In order to correctly account for Lorentz indices, one should consider the square of the operator (which, in fact, descends from the second variational derivative of the action with respect to $h_{\mu \nu}^{T T}$ ). In particular, we have an explicit expansion according to 


$$
\begin{aligned}
& h^{T T \mu \nu}(\hat{\square}-2 \hat{C})^{2} h_{\mu \nu}^{T T} \\
& \quad=h_{\mu \nu}^{T T}\left(\frac{g^{\mu \rho} g^{\nu \sigma}+g^{\mu \sigma} g^{\nu \rho}}{2} \hat{\square}-2 C^{\mu \rho \nu \sigma}\right)^{2} h_{\rho \sigma}^{T T},
\end{aligned}
$$

where also the matrix square in the pair of indices is understood, so, for instance, $\left(C^{\mu \rho \nu \sigma}\right)^{2}=C^{\mu}{ }_{\left(\alpha^{\nu}{ }_{\beta)}\right.} C^{\alpha \rho \beta \sigma}$. We wrote (29) in its expanded form to recall that the matrix multiplication must be performed with objects explicitly symmetric in the pair of indices $(\alpha, \beta)$ because they are understood at any time to act on symmetric fluctuation fields $h_{\alpha \beta}^{T T}$. We stress that in our expression (28), we used the determinants (and corresponding traces) in spaces of completely unconstrained fluctuations of spin-1 and spin-2. In their report on conformal supergravity, Fradkin and Tseytlin [16] give the following expression for the partition function:

$$
Z_{1-\text { loop }}^{2}=\frac{\operatorname{det}_{1}^{3} \hat{\square}}{\operatorname{det}_{2 T}^{2}(\hat{\square}-2 \hat{C})} .
$$

To find a relation between the two expressions for the oneloop partition function, one can derive a formula,

$$
\operatorname{det}_{2}(\hat{\square}-2 \hat{C})=\operatorname{det}_{2 T}(\hat{\square}-2 \hat{C}) \cdot \operatorname{det}_{0} \hat{\square},
$$

which is valid on any Ricci-flat background by using the trace-free property of the Weyl tensor $\hat{C}$ and of traceless perturbations $h_{\mu \nu}^{T}$. However, we remark that the above relation has nothing to do with zero modes. With the formula (31), we find a full agreement of our expression (28) with the original one due to Fradkin and Tseytlin. Finally, one can see that our expression (28) correctly predicts 6 perturbative degrees of freedom. This can be checked by setting $\hat{C}=0$ in (28) and using the counting of d.o.f. as outlined in the formula (25).

\section{B. RG flow on MSS and Ricci-flat backgrounds}

We can now go back to the discussion of the FRG flow equation for the QWG. It is clear that for the rhs of the flow equation (20), we will use effective actions (and ensuing second variations) extracted from the one-loop partition functions of QWG derived in the preceding subsection. As for the lhs of Eq. (20), we will employ the truncation ansatz to define the $\beta$ functional of the theory described by $\partial_{t} \Gamma_{L, k}$ (an additional subscript " $L$ " reminds that we work with the lhs of the flow equation). For the action $\Gamma_{L}$, we choose the most general possible truncation ansatz with three invariants containing precisely four derivatives of the metric tensor [Eqs. (3), (4), and $R^{2}$ invariant]. We stress that the action (1) of the QWG is a subcase of this truncation. The aforementioned ansatz is motivated by the structure of possible perturbative UV divergences, which can be met at the one-loop level in $d=4$ in a generic HDQG theory with four metric derivatives. On the lhs of (20), the coefficients of respective terms do contain an explicit dependence on the scale $k$. In general, the $\beta$ functional of the theory has, within our truncation ansatz, the form,

$$
\beta_{R} R^{2}+\beta_{C} C^{2}+\beta_{E} E
$$

where the $\beta$ functions are defined in a standard way as RG time $t$ derivatives of the running coupling parameters $\omega_{i}=\omega_{i}(k)$. (We have in the logarithmic RG coordinate $t=\log k / k_{0}: \beta_{i}=\partial_{t} \omega_{i}=k \partial_{k} \omega_{i}$.) These couplings appear in front of the corresponding quadratic in curvature terms in the truncation ansatz $\Gamma_{L, k}$. Let us now discuss the $\beta$ functional for the above two relevant classes of Bach-flat backgrounds.

a) Maximally symmetric spaces-We recalled that they are defined so that the Riemann tensor is

$$
R_{\mu \nu \rho \sigma}=\frac{\Lambda}{d-1}\left(g_{\mu \rho} g_{\nu \sigma}-g_{\mu \sigma} g_{\nu \rho}\right) .
$$

Particularly, in $d=4$, this gives $R_{\mu \nu \rho \sigma}^{2}=\frac{8}{3} \Lambda^{2}, R_{\mu \nu}=\Lambda g_{\mu \nu}$, so $R_{\mu \nu}^{2}=4 \Lambda^{2}$ and $R=4 \Lambda$, so $R^{2}=16 \Lambda^{2}$. With these relations, the Gauss-Bonnet term $E=\frac{8}{3} \Lambda^{2}$ and $C^{2}=0$. The corresponding $\beta$ functional (32) evaluated on this background then takes the form,

$\left.\left(\beta_{R} R^{2}+\beta_{C} C^{2}+\beta_{E} E\right)\right|_{\mathrm{MSS}}=16 \Lambda^{2}\left(\beta_{R}+\frac{1}{6} \beta_{E}\right)$.

Therefore, the only combination of $\beta$ functions that can be extracted in this case is $\beta_{R}+\frac{1}{6} \beta_{E}$.

b) Ricci-flat manifolds-They are defined so that $R_{\mu \nu}=0$, and hence $R=0$. This, in turn, implies that $R_{\mu \nu \rho \sigma}^{2}=C^{2}=E$. The $\beta$ functional thus takes the form,

$$
\left.\left(\beta_{R} R^{2}+\beta_{C} C^{2}+\beta_{E} E\right)\right|_{R_{\mu \nu}=0}=\left(\beta_{C}+\beta_{E}\right) R_{\mu \nu \rho \sigma}^{2} .
$$

The only combination of $\beta$ functions that can be obtained in this case is $\beta_{C}+\beta_{E}$.

The above two backgrounds are indeed Bach-flat in $d=4$; i.e., they are vacuum solutions of the Bach equation (8). In fact, one can make an even stronger statement, namely that the two backgrounds are vacuum solutions of the theory,

$$
S_{4 d}=\int d^{4} x \sqrt{g}\left(\alpha_{R} R^{2}+\alpha_{\mathrm{Ric}} R_{\mu \nu}^{2}\right),
$$

for arbitrary values of the parameters $\alpha_{R}$ and $\alpha_{\text {Ric }}$. This is because when $\Lambda=$ const in Eq. (33) (including $\Lambda=0$ case, so Ricci-flat case) then $\nabla_{\alpha} R_{\mu \nu}=0$ (so the Ricci tensor is covariantly constant) and also $\nabla_{\alpha} R=0$ (so the Ricci scalar is covariantly constant too). Hence, in deriving the EOM from (36), one can concentrate only on terms containing 
curvatures and no covariant derivatives. The actual proof can be found in Sec. A of SM [52].

\section{FRG flow: Some general considerations}

The quadratized action, reproducing precisely a oneloop partition function, in QWG takes the following general form:

$$
S_{\text {quad }}=\int d^{4} x \sqrt{g} \sum_{i} \phi_{i} K_{i} \phi_{i},
$$

which shows diagonality in the space of different field fluctuations $\phi_{i}$. Collectively, by $\phi_{i}$, we denote all various fluctuation fields (possible to choose selectively out of the set $\left.\left\{h_{\mu \nu}^{T T}, h_{\mu \nu}^{T}, h_{\mu \nu}, v_{\mu}^{T}, v_{\mu}, \sigma\right\}\right)$. The kinetic operators $K_{i}$ are read from the kernel of determinants of the expression for the one-loop partition functions Eqs. (24) and (28).

We note a few things here. First is that these kernels $K_{i}$ are differential operators containing two, four, or six covariant derivatives with respect to the background, in each specific case. In respective cases, these differential operators are shifted by some constant vector bundle endomorphism (proportional to the $\Lambda$ parameter and to the identity matrix II in the case of MSS) or by the Weyl tensor $\hat{C}$ in the matrix sense (for Ricci-flat background). Secondly, the actions (37), each for the case of specific backgrounds, reproduce exactly the one-loop partition functions according to the formula,

$$
Z_{1-\mathrm{loop}}^{2}=\operatorname{det}^{-1}\left(\frac{\delta^{2} S_{\mathrm{quad}}}{\delta \phi_{i}^{2}}\right),
$$

where we also used the fact that the second variational derivative (Hessian) is diagonal in the space of different fluctuations. The key point about this formula is that here we do not make any fixing of gauge symmetries, no FP determinant is needed, and there is no Jacobian of change of variables. The determinant of the Hessian to get the partition function is taken plainly without any complicacy related to gauge symmetries in general. Here, we use only physical fields as the benefit of using York decomposition. Technically, the determinant in Eq. (38) is taken in the same way as if the fluctuation fields were scalars, without any special symmetry, which would call for a modification of the functional integral prescription. Moreover, the Hessian as the operator here is clearly nondegenerate and without zero modes, hence taking its functional determinant does not create any problem.

Finally, we comment on the issue of factors in the partition functions (24) and (28) appearing both in numerators and denominators. In principle, for standard scalar particles, we have a contribution to the partition function at the one-loop level only in denominators. However, as we will show below, it does not pose any problem that we have operatorial factors also in the numerators, although an interpretation in terms of standard particles is missing here (they cannot be identified neither with phantoms, nor ghost particles). The factors in numerators can be understood as effects of the presence of local gauge symmetries in the system being preserved by the quantization process. They can be interpreted as quantum account of constraints since they effectively decrease the number of d.o.f. of the theory. Additionally, if these factors in the numerators are considered separately, then the one-loop partition function as the generalized Gaussian integral of the operator, is not convergent even in the Euclidean setting. We emphasize that in the form of the partition functions, as found in Eqs. (24) and (28), we do not see any explicit dependence on the Weyl coupling $\omega_{C}$; hence, this will not show up anywhere in the action (37) nor in the rhs of the FRG flow equation (20). Quite generally, any overall factors (and in particular their signs) of terms in the quadratized action (37) are irrelevant for taking the Hessian and the ensuing functional determinants.

We factorize each of the kernel factors $K_{i}$ in (37) to monomials containing precisely two derivatives, so to monomials containing only one power of the box operator in a corresponding representation. This we make according to the formula valid for any $i$,

$$
K_{i}=\prod_{j}\left(\hat{\square}-Y_{i, j}\right)^{ \pm 1}
$$

One can then rewrite the quadratized action (37) in the form,

$$
S_{\text {quad }}=\int d^{4} x \sqrt{g} \sum_{i} \sum_{j} \phi_{i}\left(\hat{\square}-Y_{i, j}\right)^{ \pm 1} \phi_{i},
$$

where the shifts $Y_{i, j}$ are of the general form as explained above. The power exponents \pm 1 should be chosen according to whether the factor is to be placed in the denominator or in the numerator of the partition function in Eqs. (24) and (28). One can convince oneself that this form of the action reproduces again the correct form of the one-loop partition functions on each background, but now the advantage is that all kinetic operators carry only two derivatives.

Furthermore, we now discuss the issue of the scale dependent wave function renormalization for all fields involved in the construction of the partition functions [so also appearing in their generating actions (40)]. The wave function renormalization is obtained via the following transformation:

$$
\phi_{i} \rightarrow Z_{k, i}^{1 / 2} \phi_{i}
$$

The renormalization factors $Z_{k, i}$ are in the case of gauge theory with one coupling $\omega_{C}$ strictly related since the quantum dynamics of fluctuations is governed by the same 
action term [here, the action of QWG in (1)]. Since these fields are in the same gauge symmetry multiplet, they have the same wave function renormalization factor $Z_{k}^{1 / 2}$.

In order to take a truncation ansatz for the running effective action $\Gamma_{R, k}$ on the rhs of the FRG flow equation (20) that faithfully reproduces RG effects (related in particular to anomalous dimensions of quantum fields), we must take into account the wave function renormalization effects on fluctuations. This amounts to substitution of renormalized fields into the generating action (40) as the arguments. Therefore, we take the following RG-improved truncation ansatz for $\Gamma_{R, k}$ :

$$
\begin{aligned}
\Gamma_{R, k} & =S_{\text {quad }}\left[Z_{k}^{1 / 2} \phi_{i}\right] \\
& =\int d^{4} x \sqrt{g} \sum_{i} \sum_{j} \phi_{i} Z_{k}\left(\hat{\square}-Y_{i, j}\right)^{ \pm 1} \phi_{i} .
\end{aligned}
$$

We construct the IR-cutoff action in a standard Wilsonian way. Namely, we use the one-loop partition function generating action (40), and we modify each quadratic in derivatives kinetic term by adding a suitably chosen IR-cutoff kernel function $R_{k}$. Consequently, we obtain the IR-cutoff action in the form,

$$
\Delta S_{\mathrm{IR}}=\int d^{4} x \sqrt{g} \sum_{i} \sum_{j} \phi_{i}\left(\hat{\square}-Y_{i, j}+R_{k, i} \hat{\mathbb{I}}\right)^{ \pm 1} \phi_{i} .
$$

The role of the IR-cutoff kernel $R_{k, i}$ is to suppress the contribution to the functional integral of the field modes corresponding to eigenvalues $\lambda_{n}$ smaller than the cutoff scale $k^{2}$ (the so-called low energy modes). This cutoff kernel is a function of the operator, which describes the dynamics of modes. We will start with the cutoff kernel $R_{k, i}=R_{k, i}(\hat{\square})$ with the $\hat{\square}=\hat{\Delta}$ operator. For technical convenience, we choose as a "cutoff profile", the so-called Litim cutoff function (or optimized cutoff); see Eq. (38) from [52]. In principle, each different subspace of fluctuation fields $\phi_{i}$ might have its own cutoff function $R_{k, i}$. However, for simplicity, we will choose them to be identical and given by one universal function $R_{k}$. Let us also remark that we do an IR suppression of modes (in the Wilsonian spirit) also for factors which appear in the numerator of the partition functions. This is allowed by the versatility of the functional RG methods and the flexibility of the flow equation (20). Then the regularized kinetic operator for all modes is given by

$$
Z_{k}\left(\hat{\square}+R_{k}(\square) \hat{\Pi}+Y_{i, j}\right)_{\phi_{i}} \cdot
$$

Now, we can write down the Hessian operator $\Gamma_{R}^{(2)}$, which is used in the rhs of the FRG flow equation (20). Taking second variational derivatives results in stripping the fluctuations from both sides of the quadratized action (40). With this simplification in mind, we can write schematically

$$
\Gamma_{R}^{(2)}=\prod_{i} \otimes \prod_{j} Z_{k}\left(\hat{\square}-Y_{i, j}\right)_{\phi_{i}}^{ \pm 1}
$$

where, in each subspace of fluctuations $\phi_{i}$, we distinguish the operator by putting a mark of the subspace in the subscript after it. Similarly, we find that the regularized Hessian is the operator $\Gamma_{R}^{(2)}+R_{k}$ in (20) that has a schematic representation,

$$
\Gamma_{R}^{(2)}+R_{k}=\prod_{i} \otimes \prod_{j} Z_{k}\left(\hat{\square}+R_{k}(\square) \hat{\mathbb{I}}-Y_{i, j}\right)_{\phi_{i}}^{ \pm 1} .
$$

Eventually, based on the above formula and Eq. (20), the FRG flow equation takes the form,

$$
\partial_{t} \Gamma_{L, k}=\frac{1}{2} \sum_{i} \sum_{j} \pm \operatorname{Tr}_{\phi_{i}}\left(\frac{\left(\partial_{t} R_{k}-\eta R_{k}\right) \hat{\mathbb{I}}}{\hat{\square}+R_{k} \hat{\mathbb{I}}-Y_{i, j}}\right),
$$

where we have defined the anomalous dimension (identical for all fluctuation fields) as $\eta=\partial_{t} \log Z_{k}$. Moreover, the \pm signs depend on what was in the exponent on the corresponding term in the Hessian (45) or in other words, whether the factor was originally in the denominator or the numerator of the partition function, respectively. The ultimate correctness of the above steps that lead to the FRG flow equation (47), will be verified below by a number of independent checks.

Finally, we wish to discuss our choice of the minimal consistent ansatz for the effective action appearing on the lhs of the flow equation (47). This object is denoted by $\Gamma_{L, k}$, and it contains explicit dependence on the scale $k$ through the overall running couplings. As explained in the formula (32), the most general truncation for the lhs may contain three terms quadratic in curvatures, each leading to terms with four derivatives of the metric. However, we will project our resulting RG flow onto a smaller subspace without the $R^{2}$ term. This is motivated by the fact that at the one-loop approximation in QWG, the $\beta$ function for the $R^{2}$ term is exactly zero. In this perturbative scheme, one can show that when the proper care is taken and the conformal symmetry is preserved on the quantum level of computation of UV divergences (and hence, in the computation of the perturbative one-loop effective action), then the ensuing $R^{2}$ divergences are not generated at all [16]. This result can be seen as a (partial) self-protection of conformal symmetry on the quantum level, since the only acceptable UV divergences are absorbed by the conformally covariant counterterm $C^{2}$ and the topological one $E$. It is expected that the $R^{2}$ divergences will show up at the two-loop level. Some partial computation in this direction was presented in [18]; however, the results are not fully conclusive, and one might still entertain some hope that the conformal symmetry is powerful enough to prevent appearing of such nonconformal $R^{2}$ divergences also at two loops or even at a 
higher loop level. The arguments against this hope (apart from the aforementioned partial two-loop computation in [18]) are mostly related to the issue of conformal anomaly (CA) [79]. However, one can try to waive them pointing to the issue of the ambiguities of CA [80].

Already at the one-loop level, despite the need for covariantly and only conformally looking counterterms, the obstacle for full quantum conformality is the presence of $\mathrm{CA}$, which is there due to nonvanishing $\beta$ functions of the theory, namely $\beta_{C} \neq 0$ and $\beta_{E} \neq 0$. The theory is with divergences and there are perturbative $\beta$ functions; hence, the CA is nonvanishing. And since in the QWG, the conformal symmetry is in the local (gauged) version, then the fact that $\mathrm{CA}$ is nonzero spoils the conformal symmetry on a quantum level (in particular, it destroys conformal Ward identities) basically the same way like gauge anomalies spoil gauge symmetries in Yang-Mills theories; therefore, these anomalies have to be avoided at all cost. For consistent local conformal theory on a quantum level, we would need to have full cancellation of all UV divergences; hence, the theory should be UV finite and hence, CA free. Till these days, only two classes of such theories including quantum gravitation are known. First are superconformal anomaly free theories obtained by Fradkin and Tseytlin in the $\mathcal{N}=4$ conformal supergravity models. Second are recently found perturbatively UV-finite quantum gravitational theories [35,81-84] considered as an extension of superrenormalizable higher derivative theories.

The situation with QWG without supersymmetry, without other matter species, and without higher-curvature operators needed to give UV finiteness in [81] is that probably the conformal symmetry is not strong enough to constrain the quantum dynamics at higher loop orders. And starting from two loops on the $R^{2}$, divergences are generated, and the conformal symmetry is completely washed out by quantum corrections. We are sure in having conformal symmetry on the classical tree level and also partially on the one-loop level. Since couplings in the theory are asymptotically free (more on this in Sec. IV B) and the theory is weakly coupled at high energies, then the problem of the conformal anomaly is a problem for UV completion of the theory. Our take on the issue of CA is that the conformal symmetry is broken at low-enough energies, and this is closely related to the dynamical breakdown of scale invariance in the IR sector of the theory. To explore this point more, we will mainly focus on FRG flow in the IR sector.

The fact that $\beta_{R}=0$ at one-loop in QWG is quite miraculous, but on more general level, this suggests that a natural scheme should be used, for example, in nonperturbative FRG, in which $\beta_{R}$ is parametrically smaller than other nonperturbative $\beta$ functions of the system. It is expected that at the perturbative two-loop level, the $\beta_{R}$ is expressed through higher inverse powers of the Weyl coupling $\omega_{C}$ of the theory. Therefore, exploiting this hierarchy of $\beta$ functions, we can assume the following ansatz for $\Gamma_{L, k}$ (cf. [85]):

$$
\Gamma_{L, k}=\int d^{4} x \sqrt{g}\left[\omega_{C}(k) C^{2}+\omega_{E}(k) E\right],
$$

where we have neglected the $R^{2}$ term and its running coupling $\omega_{R}=\omega_{R}(k)$. The choice of this ansatz for $\Gamma_{L, k}$ will allow us to read unambiguously two $\beta$ functions, according to the Eq. (32). By adopting the two backgrounds discussed above (MSS and Ricci-flat), we can read off $\beta_{E}$ and $\beta_{C}$. This will be done in Sec. IV. One notices a difference in sign in front of the $\omega_{C}$ coupling in Eq. (48) compared with Eq. (1) and conventions stipulated there in Sec. II. This is the effect of performing a Wick rotation to the Euclidean signature, while both types of couplings $\omega_{C}$ and $\alpha$ are always required to be positive.

In order to explicitly compute traces involved in (47), we will employ the heat kernel technique outlined in Supplemental Material (SM) [52]. In particular, we use the formula (35) from [52],

$$
\operatorname{Tr} f(\Delta)=\frac{1}{(4 \pi)^{d / 2}} \sum_{n=0}^{+\infty} Q_{\frac{d}{2}-n}[f] B_{2 n}(\Delta),
$$

where we restrict ourselves to two cases: $n=2$ and $d=4$. This choice is due to the fact that we want to project the rhs of the FRG flow equation onto the subspace spanned by the four-derivative terms present in the truncation ansatz $\Gamma_{L, k}$ (48). Moreover, let us notice that the heat kernel coefficients $B_{2 n}(\Delta)$ contain exactly only terms with $2 n$ derivatives of the metric tensor (cf. Sec. C of SM [52]). As the operators $\Delta$, we take in each case $\Delta=\hat{\square}+\hat{Y}$, which is a two-derivative operator, possibly shifted by some endomorphism $\hat{Y}$ of the internal vector bundle (it acts there as a matrix multiplication, not as a differential operator). The general IR-regulated (Euclidean) propagator of modes will have the structure,

$$
G_{k}(z)=\frac{1}{z+R_{k}(z)+\varpi}
$$

where we identify $z \equiv \hat{\square}$ as the main argument here and the shifts $\varpi$ (acting effectively like masses) are identified as $\varpi=Y_{i, j}$.

\section{ANALYSIS OF $\beta_{C}$ AND $\beta_{E}$ FUNCTIONS}

\section{A. System of two $\beta$ functions}

Let us now use the enhanced one-loop relations for two $\beta$ functions $\beta_{C}$ and $\beta_{E}$ [implied by the ansatz (48)] in the form, 


$$
\begin{gathered}
\beta_{E}=\frac{1}{2}(2-\eta)\left[-\frac{21}{40}\left(1-\frac{\frac{2}{3} \Lambda}{k^{2}}\right)^{-1}+\frac{9}{40}\left(1-\frac{\frac{4}{3} \Lambda}{k^{2}}\right)^{-1}\right. \\
-\frac{179}{45}\left(1+\frac{\Lambda}{k^{2}}\right)^{-1}-\frac{59}{90}\left(1+\frac{\frac{1}{3} \Lambda}{k^{2}}\right)^{-1} \\
\left.+\frac{479}{360}\left(1+\frac{2 \Lambda}{k^{2}}\right)^{-1}-\frac{269}{360}\left(1+\frac{\frac{4}{3} \Lambda}{k^{2}}\right)^{-1}\right], \\
\beta_{C}+\beta_{E}=\frac{2-\eta}{2} \frac{137}{60},
\end{gathered}
$$

with the anomalous dimension of the graviton field,

$$
\eta=-\frac{1}{\omega_{C}} \beta_{C}
$$

Here, $\omega_{C}=\omega_{C}(k)$ represents a running coupling parameter in front of the $C^{2}$ term in the action (1)-the so-called Weyl coupling. Explicit derivation of the results (51)-(53), including computation of $\eta$ up to one loop, can be found in Secs. D and E of SM [52].

The above two $\beta$ functions $\beta_{C}$ and $\beta_{E}$ follow from the functional RG, and therefore, we can say that they are RG improved because they include quantum effects related to both the threshold phenomena and nontrivial anomalous dimension of quantum fields. Let us recall that we have projected the full functional RG flow (20) onto a subspace of couplings consisting of more than just one Weyl coupling $\omega_{C}$. We have included also the induced effect on the running of the coupling $\omega_{E}$, while the effects on $\omega_{R}$ were neglected due to the hierarchy in the system of $\beta$ functions, first found at the one-loop level approximation, but expected to hold also for higher loops (or even nonperturbatively). We notice that the effects of threshold phenomena show up explicitly only in the expression (51) for $\beta_{E}$. However, due to the relation in (52), the solution for $\beta_{C}$ will also contain the threshold factors. Finally, we observe that the effect of an anomalous dimension $\eta$ enters only multiplicatively in the system of $\beta$ functions (51)-(52). This will have simplifying consequences when we will search for the FP's of the coupled system, both in the UV as well as in the IR limit.

Let us now briefly discuss the reasons for the appearance of threshold phenomena in our system. As it is seen from the expressions for the one-loop partition functions Eqs. (24) and (28), the box-kinetic operator of modes is shifted in such a way as to produce IR thresholds, only in the case of MSS background. The shift by a matrix of a Weyl tensor $\hat{C}$ on the Ricci-flat background does not generate any threshold because the Weyl tensor is completely traceless in each pair of its indices. These shifts on MSS are analogous to massive modes in standard QFT analyzed on flat spacetime. They effectively slow down the RG flow in the IR regime because the quantum fields become heavy. As it is well-known on MSS [especially on anti-de Sitter (AdS) spacetime with a Minkowski signature], there exist bounds on the masses of "healthy" modes that can be considered in QFT. The dynamics of modes with mass square parameters smaller than the so-called Breitenlohner-Freedman (BF) bound put the modes in danger regarding the unitarity of the theory, basically the same way like tachyons endanger stability of flat spacetime QFT. These BF bounds depends naturally on the spin of modes and also on the $\Lambda$ parameter of the MSS spacetime; see Eq. (33). Moreover, reaching the BF bound corresponds to swapping all the modes from massive to massless; hence, some enhancement of symmetries of the theory might be expected. The BF bound is the boundary dividing healthy from unhealthy modes. In this connection, one can pose an interesting question, namely if in our expression (24) for the one-loop partition function, we do not have effective masses which are below the BF bound. However, close inspection of (24) reveals that we have modes in danger (with negative coefficient in front of $\Lambda$ parameter) only in the two factors belonging to the spin-2 traceless modes subsector. All other modes with scalar and vector characters are healthy. It is important to note that this discussion is purely academic here since for the issue of RG flow in the Euclidean signature the presence of modes below the BF bound will be completely inessential. Moreover, we would like to analyze the FRG flows on the energy scales ranging from UV regime $(k \rightarrow+\infty)$ to IR $(k \rightarrow 0$ in Euclidean), so for our purposes, this will be more than enough, and we do not have to worry here about violation of the BF bound and its physical effects.

One might be deluded by the superficial simplicity of the system of equations (51)-(52) and think that by simple subtraction of (51) from (52), one gets already a full solution for $\beta_{E}$ and $\beta_{C}$. This is true only in a simple case, when the anomalous dimension $\eta$ is neglected. In general, however, one should pay attention to the dependence of $\eta$ on $\omega_{C}$ and $\beta_{C}=\partial_{t} \omega_{C}$, which appears both in (51) and in (52). The system is still possible to be disentangled algebraically for the two $\beta$ functions. In passing we note that, as it is common with RG (global) systems, this is still a system of ordinary differential equations (ODE's) for running coupling parameters, here, respectively, for $\omega_{C}$ and $\omega_{E}$. We will not solve explicitly these ODE's for couplings here. We will just concentrate on the FP's of this system.

By solving algebraically the above system of implicit equations, we obtain

$$
\beta_{C}=\frac{b-X}{1+y(X-b)}, \quad \beta_{E}=\frac{X}{1+y(X-b)},
$$

where 


$$
\begin{aligned}
X= & -\frac{21}{40}\left(1-\frac{\frac{2}{3} \Lambda}{k^{2}}\right)^{-1}+\frac{9}{40}\left(1-\frac{\frac{4}{3} \Lambda}{k^{2}}\right)^{-1} \\
& -\frac{179}{45}\left(1+\frac{\Lambda}{k^{2}}\right)^{-1}-\frac{59}{90}\left(1+\frac{\frac{1}{3} \Lambda}{k^{2}}\right)^{-1} \\
& +\frac{479}{360}\left(1+\frac{2 \Lambda}{k^{2}}\right)^{-1}-\frac{269}{360}\left(1+\frac{\frac{4}{3} \Lambda}{k^{2}}\right)^{-1},
\end{aligned}
$$

with $b=137 / 60$ and $y=1 / \omega_{C}$. One can easily convince oneself that the origin of the common denominator $1+$ $y(X-b)$ is entirely due to inclusion of the effect of anomalous dimension $\eta$. In the case when $\eta$ is neglected, the latter boils down to unity. Alternatively, this limit corresponds to taking a regime, in which the Weyl coupling $\omega_{C}$ takes large values $\left(\omega_{C} \rightarrow \infty\right.$, so $\left.y \rightarrow 0\right)$, i.e., the regime in which the theory is very well described perturbatively (in terms of the coupling $\alpha$ ). When one decides to neglect these common denominators, one is left with the simplified expressions of the form,

$$
\beta_{C}=b-X, \quad \beta_{E}=X,
$$

which as we will see shortly, are sufficient to cast light on the issue of existence of FP's of the FRG flow, and yet include the effect of threshold phenomena. Should we have removed the threshold phenomena from our description, the system would acquire the form of one-loop perturbative $\beta$ functions as derived in [16] for QWG in a dimensional-regularization scheme, cf. Eqs. (76) and (92) from SM [52]. As a matter of fact, all the threshold phenomena are included in the expression called $X$. When one takes the limit $\Lambda / k^{2}$ to zero, then all threshold factors are removed, and this expression just reduces to a number $X=\beta_{E}^{\mathrm{FT}}=-87 / 20$.

\section{B. UV FP of the system of $\boldsymbol{\beta}$ functions}

As already known from the seminal papers $[86,87]$, the system of $\beta$ functions (51) and (52) reaches a trivial Gaussian FP in the UV regime. When $\kappa=k / \sqrt{|\Lambda|} \gg 1$, the threshold effects are completely inessential and can be neglected, cf. Eqs. (51) and (74) from SM [52]. Irrespective of the initial values of the couplings $\omega_{C 0}=\omega_{C}\left(t_{0}\right)$ and $\omega_{E 0}=\omega_{E}\left(t_{0}\right)$, the leading behaviors of the RG running in the $\mathrm{UV}$ (for $t \gg 1$ ) is $\omega_{C} \sim t \beta_{C}^{\mathrm{FT}}$ and $\omega_{E} \sim t \beta_{E}^{\mathrm{FT}}$. This means that the absolute values of the couplings must necessarily grow in the UV regime (they decrease in the actual negative values of $\omega_{E}$ coupling). In the same vein, it might be argued that in the UV regime one can also neglect the anomalous dimension $\eta$, cf. Eq. (53). Thus, for the UV running, it suffices to use only the nonimproved one-loop perturbation results (76) and (92) in SM [52].

All these arguments are self-consistent and lead to the conclusion that the UV FP inevitably exists and realizes the asymptotic freedom (AF) scenario (in much the same way as in non-Abelian gauge theories). Since our perturbation analysis is carried out in terms of the coupling $\alpha^{2} \propto 1 / \omega_{C}$, the fact that in the UV, $\omega_{C} \rightarrow+\infty$ bolsters even more the correctness of our one-loop results. Actually near the UV Gaussian FP, it is the coupling $\alpha$ that goes to zero.

\section{IR FP's from the system of $\boldsymbol{\beta}$ functions}

Let us now come back to the issue of IR FP's of the system. We will shortly see that the inclusion of threshold phenomena, which are present in any mass-dependent renormalization scheme, is of crucial importance in our analysis. In fact, should we have studied only the simplified system of $\beta$ functions (76) and (92) in SM [52], we would not find any interesting behavior of the RG flow in the IR (similarly to the case of QCD where the coupling grows stronger and gets out of the perturbative regime). There are no any IR FP's in such a simplified scheme. To look for some less trivial behavior in the IR, we must thus include some additional nonperturbative effects. Here, this feature is brought about by our usage of FRG and account of IR decoupling of massive modes.

In order to look for the FP's of the system in the IR, we must solve equations $\beta_{C}(k)=0$ and $\beta_{E}(k)=0$. Already here, one can see a huge simplification because in order to find zeros, we do not need to solve the full system (54). Actually, we can completely forget the denominators and solve only Eqs. (56), where factors $\Lambda / k^{2}$ are taken into account. This signifies that the anomalous dimension $\eta$ does not influence the location of the IR FP's within the limits implied by our truncation of FRG.

Direct numerical solutions of the equations $\beta_{C}(\kappa)=0$ and $\beta_{E}(\kappa)=0$ reveal that they are both satisfied at (approximately) simultaneous values of $\kappa=k / \sqrt{|\Lambda|}$; see also Fig. 1. This is a smoking gun for the fixed point. Actual numerical values for the critical energy scales $\kappa_{c}$, at which the corresponding $\beta$ functions cross zero, are, respectively,

$$
\kappa_{c, C} \approx 1.17709 \text { and } \kappa_{c, E} \approx 1.19163,
$$

for the MSS with $\Lambda>0$, and

$$
\kappa_{c, C} \approx 1.49722 \text { and } \kappa_{c, E} \approx 1.52128,
$$

for the MSS with $\Lambda<0$. The zeros (57) and (58) are automatically zeros of the system (54). One sees that the location of energy scales is almost identical (up to $2 \%$ accuracy) for the couplings $\omega_{C}$ and $\omega_{E}$ for both $\Lambda>0$ and $\Lambda<0$. We expect that the inclusion of higher loop effects or extension of our truncation ansatz will make this discrepancy even smaller, such that in an exact fully nonperturbative theory, the locations of two zeros coalesce into the one unique location of a genuine FP for both couplings. Below we perform a general analysis of the situation near critical energy scale $\kappa_{c}$ for a general 


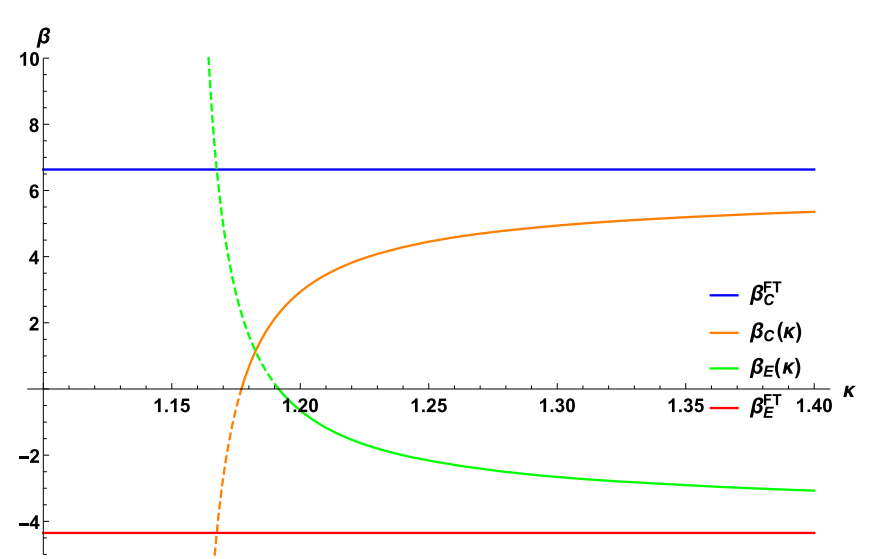

FIG. 1. FRG $\beta$ functions for the running couplings $\omega_{C}$ and $\omega_{E}$ in dependence on the dimensionless scale $\kappa=k / \sqrt{|\Lambda|}$. We depict the situation with $\Lambda>0$. The superscript "FT" denotes the Fradkin-Tseytlin $\beta$ functions [16]. Both $\beta_{C}(\kappa)$ and $\beta_{E}(\kappa)$ asymptotically approach $\beta_{C}^{\mathrm{FT}}$ and $\beta_{E}^{\mathrm{FT}}$, respectively, in the deep UV regime. Running $\beta$ functions reach their zero values in the IR region at approximately identical scales $\kappa$, cf. Eqs. (57)-(58). Note that at respective critical scales $\kappa_{c}$ both $\beta$ functions have finite values of the slope parameters $\left.\partial_{\kappa} \beta(\kappa)\right|_{\kappa=\kappa_{c}}$. Dashed lines are used to denote (unphysical) extensions of the running $\beta$ functions past the zero point. For simplicity's sake, we do not assume here any contribution from the anomalous dimension $\eta=0$ implying $y=0$.

coupling $\omega$. The specification whether this is $\omega_{C}$ or $\omega_{E}$ will be important only for the numerical values that we quote at the end.

Now, from Fig. 1, we see that the slope parameter at the zero crossing $\left.\partial_{\kappa} \beta(\kappa)\right|_{\kappa=\kappa_{c}} \equiv a$ is finite and positive for $\beta_{C}$ and negative for $\beta_{E}$. So, we can Taylor expand the $\beta$ function $\beta(\kappa)$ around $\kappa_{c}$, so that

$$
\beta(\kappa)=a\left(\kappa-\kappa_{c}\right)+a_{2}\left(\kappa-\kappa_{c}\right)^{2}+\cdots .
$$

In what follows, we will concentrate only on the effects of the first term in the above Taylor expansion (with the coefficient $a$ of the first derivative), while the more refined analysis, which includes also the second derivative coefficient $a_{2}$ is presented in Sec. F of SM [52].

It is easy to rewrite the expansion in Eq. (59) in terms of coupling $\omega$. First, from (59), we get

$$
\omega(\kappa)-\omega_{*}=a\left(\kappa-\kappa_{c}\right)-a \kappa_{c} \log \frac{\kappa}{\kappa_{c}}+\cdots,
$$

where $\omega_{*}=\omega\left(\kappa_{c}\right)$. Relation (60) can be inverted so that we have

$$
\kappa=-\kappa_{c} W\left(-\exp \left[-\frac{\omega-\omega_{*}}{a \kappa_{c}}-1\right]\right)+\cdots
$$

where $W$ is a Lambert function. At this stage, we should recall that a Lambert function is a double valued on the interval $(-1 / e, 0)$. Since $\kappa / \kappa_{c} \geq 1$, the $W(\ldots) \leq-1$, and we should work with the lower branch of the Lambert function known as $W_{-1}(\ldots)$. For small $\left(\omega-\omega_{*}\right) /\left(a \kappa_{c}\right)$ [which for both $\omega_{C}$ and $\omega_{E}$ is positive, cf. Eq. (60) and actual numerical values quoted in Eqs. (73) and (74) below], we can expand the rhs of (61). This gives

$$
\begin{aligned}
\kappa= & -\kappa_{c} W_{-1}\left(-\exp \left[-\frac{\omega-\omega_{*}}{a \kappa_{c}}-1\right]\right) \\
= & \kappa_{c}+\sqrt{\frac{2 \kappa_{c}}{a}\left(\omega-\omega_{*}\right)}+\frac{2}{3 a}\left(\omega-\omega_{*}\right) \\
& +\mathcal{O}\left(\left(\omega-\omega_{*}\right)^{3 / 2}\right),
\end{aligned}
$$

and so the $\beta$ function (59) reads

$$
\beta=\sqrt{2 a \kappa_{c}\left(\omega-\omega_{*}\right)}+\frac{2}{3}\left(\omega-\omega_{*}\right)+\cdots
$$

Note that the result (63) holds true both for $\beta_{C}$ and $\beta_{E}$, since the product $a\left(\omega-\omega_{*}\right) \geq 0$ in both cases.

The fact that the $\beta$ function can develop at a finite $\mathrm{RG}$ scale $\kappa=\kappa_{c}$, a nonanalytic behavior of the type (63) is well-known from holography, where it signalizes the presence of a multibranch holographic RG flow that arises due to bounce solutions in the bulk [88-90]. In such cases, the corresponding $\kappa_{c}$ is merely a turning point on the way to a genuine IR fixed point. Despite that both $\beta$ functions $\beta_{C}$ and $\beta_{E}$ turn zero at $\kappa=\kappa_{c}$, this is not a true FP of RG flow of QFT because it happens at some finite scale $\kappa_{c}$. We remind that the $\mathrm{RG}$ flow stops when two conditions are met: all beta functions vanish and the energy scale is $k=0$ (IR FP) or $k=+\infty$ (UV FP). Similarly, in the holographic (dual) perspective, the turning point at $\kappa=\kappa_{c}$ corresponds to a surface embedded in AdS-like $d=5$ geometry located at some finite radial coordinate $\rho_{c} \sim \kappa_{c}^{-1}$. Since we know that for smaller energy scales $\kappa<\kappa_{c}$, we still have quantum degrees of freedom in the theory (i.e., they were not all integrated out), then this means that on the gravitational side, gravitational evolution of the dynamical $d=5$ spacetime must also continue past the bounce point with $\rho=\rho_{c}$ towards larger values of the AdS radial coordinate. The true IR FP shall correspond to a conformal boundary of AdS at infinite values of the radial AdS coordinate.

The gravitational bounce is an example of FLRW spacetime for which the cosmological scale factor exhibits a bounce behavior. On the bulk side, such behavior of spacetime is, of course, caused by some (exotic) matter source present $[91,92]$. Typically the running of couplings in front of scalar operators is naturally described by dynamical bulk scalar fields with the particular mass parameter related to the scaling dimension of the operator on the boundary theory side, according to the AdS/CFT dictionary. When the $\beta$ function shows a nonanalytic bouncing behavior like the one in Eq. (63), then the similar behavior must also be exhibited by the corresponding bulk 
scalar field. This square-root-like singularity may mean that above the critical value $\rho_{c}$, the real profile of this bulk scalar field simply does not exist, or it becomes purely imaginary, which is however forbidden from the point of view of unitarity in QFT. On the other hand, the critical point can be interpreted as a joining point (or a bifurcation point depending on the direction of the flow) for two branches of solutions for the bulk scalar field. But here one sees that the gravitational spacetime easily extends beyond that critical radii surface with $\rho=\rho_{c}$, and one can still look for the true IR FP of the quantum system corresponding to the boundary of asymptotically AdS spacetime at $\rho \rightarrow+\infty$.

Let us note that this type of bouncing RG flow, i.e., flow that displays one or more bounces before reaching the IR $\mathrm{FP}$, is quite easy to encounter in a number of holography scenarios [88]. The bouncing RG flow of the above type has been seen also in condensed-matter effective field theories $[93,94]$.

Using now the formula (63) for the expression of the $\beta$ function near the turning FP, we can analytically extend this behavior past the turning FP to the RG scale where $0 \leq \kappa \leq \kappa_{c}$. Since in this case $\kappa / \kappa_{c} \leq 1$, we should employ in (61) the upper branch of the Lambert function, known as $W_{0}(\ldots)$. The sole effect of this step is that $\kappa$ from (62) will be smoothly taken through the turning point with $\kappa=\kappa_{c}$ to $\kappa$ of the form,

$$
\begin{aligned}
\kappa & =-\kappa_{c} W_{0}\left(-\exp \left[-\frac{\omega-\omega_{*}}{a \kappa_{c}}-1\right]\right) \\
& =\kappa_{c}-\sqrt{\frac{2 \kappa_{c}}{a}\left(\omega-\omega_{*}\right)}+\frac{2}{3 a}\left(\omega-\omega_{*}\right)+\mathcal{O}\left(\left(\omega-\omega_{*}\right)^{3 / 2}\right) .
\end{aligned}
$$

So, the key effect of the above analytical continuation is that

$$
\sqrt{\omega-\omega_{*}} \rightarrow-\sqrt{\omega-\omega_{*}}
$$

(and such a flipping of a sign is present also for all terms with higher half-integer power exponents on $\omega-\omega_{*}$ ). This is a hallmark relation stemming from the square-root-like singularity of the RG flow and strictly related to holographic bounces in the bulk description. From (64) treated as an exact relation, we get the flow of the coupling $\omega$ in the region $0 \leq \kappa \leq \kappa_{c}$, namely

$$
\omega=\omega_{*}+\frac{9 a}{2} \kappa_{c}^{1 / 3}\left[\kappa^{1 / 3}-\kappa_{c}^{1 / 3}\right]^{2} .
$$

Taking this dependence as being exact on the energy scales past the turning FP, one can find the true FP occurring at $\kappa=0$ (i.e., in the deep IR). This implies that the IR FP value of the coupling is

$$
\omega_{* *}=\omega(\kappa=0)=\omega_{*}+\frac{9}{2} a \kappa_{c} .
$$

Ensuing behavior of the $\beta$ function near $\omega_{* *}$ is given by

$$
\beta=\kappa \frac{d \omega}{d \kappa}=3 a\left(\kappa_{c} \kappa\right)^{1 / 3}\left[\kappa^{1 / 3}-\kappa_{c}^{1 / 3}\right] .
$$

All information regarding the FP's can be extracted from the set of (critical) scaling exponents that are defined in terms of the (negative) eigenvalues of the stability matrix at the FP, i.e.,

$$
\theta \in-\left.\sigma\left(\frac{\partial \beta_{i}}{\partial \omega_{j}}\right)\right|_{\omega=\omega_{* *}},
$$

where $\sigma$ denotes the corresponding spectrum and $\beta_{i}=$ $\left\{\beta_{C}, \beta_{E}\right\}$, while $\omega_{i}=\left\{\omega_{C}, \omega_{E}\right\}$. In our case of the IR FP, the only nonzero elements of the stability matrix are

$$
\left.\frac{\partial \beta_{C}}{\partial \omega_{C}}\right|_{\omega=\omega_{* *}}=-\sqrt{\frac{a \kappa_{c}}{2\left(\omega_{* *}-\omega_{*}\right)}}+\frac{2}{3}=\frac{1}{3},
$$

and similarly,

$$
\left.\frac{\partial \beta_{E}}{\partial \omega_{E}}\right|_{\omega=\omega_{* *}}=\frac{1}{3}
$$

which implies that

$$
\theta=\left\{-\frac{1}{3},-\frac{1}{3}\right\}
$$

One refers to an IR FP as IR stable, if all eigenvalues $\theta$ are negative, so, from (72), we can conclude that the FP in the IR is completely stable in the space of all considered couplings.

Critical exponents allow for a precise definition of the (conformal) scaling dimensions of the operators through the relation $\theta_{i}=d-\Delta_{i}$, where $\Delta_{i}$ is the scaling dimension associated with a given operator. In our case, the operators $\sqrt{|g|} C^{2}$ and $\sqrt{|g|} E$ (and their related couplings $\omega_{C}$ and $\omega_{E}$ ) are classically (at tree-level) marginal with their canonical dimensions $D_{i}=\left(\Delta_{i}\right)_{\mathrm{cl}}=4$. Due to quantum (loop) corrections, their quantum $\Delta_{i} \neq 4$ and the corresponding deviation from 4 , known as anomalous scaling, which is defined as $\gamma_{i}=\Delta_{i}-D_{i}$, equals $\gamma_{i}=-\theta_{i}=1 / 3$. The latter implies that two involved operators become relevant operators near the IR FP (and also the two couplings $\omega_{C}$ and $\omega_{E}$ are IR relevant).

For definiteness, we list below the numerical values of the products $9 a \kappa_{c} / 2$ that correspond to $\omega_{* *}-\omega_{*}$ [in accordance with Eq. (67)]. We also use a simplifying condition $y=0$. In particular, for the case of $\Lambda>0$, we find 


$$
\frac{9}{2} a_{C} \kappa_{c, C} \approx 1371.98, \quad \frac{9}{2} a_{E} \kappa_{c, E} \approx-511.201,
$$

while for the case of $\Lambda<0$, we have

$$
\frac{9}{2} a_{C} \kappa_{c, C} \approx 842.063, \quad \frac{9}{2} a_{E} \kappa_{c, E} \approx-490.829 .
$$

Let us recall that the value of the $\omega_{*}$ can be fixed by the initial conditions of RG flow; hence, the same level of arbitrariness will be inherited in the IR FP values $\omega_{* *}$ of the $\omega$ couplings. It is also obvious that both couplings $\omega_{C}$ and $\omega_{E}$ at IR FP are nonzero, and hence, the IR FP is nonGaussian. This is, of course, very important conclusion because the Weyl gravity turns out to be a nonperturbative theory in IR along similar lines as QCD.

It should be borne in mind that the existence of the IR FP ought to be a universal property of the system independent from particular details of the renormalization scheme used. In particular, as we emphasized above, the only crucial requirement from the renormalization scheme is its mass dependence so the fact that the contributions from IR modes are properly secured. In fact, it is always the case with computations done in the FRG framework that the precise locations of FP's do depend on characteristics of the renormalization process, but their existence or some other properties (related to critical exponents or the dimensionality of the critical surface) are universal, independent of gauge fixing choice, and renormalization details. Furthermore, in contrast to $\beta$ functions themselves (that are not observable), the aforementioned properties constitute genuine observable pieces of information that can be extracted nonperturbatively from a theory, which reaches a nontrivial FP within the FRG framework.

At this point few comments are in order:

(i) We can observe that the turning point of the RG flow obtained above arises at the finite value of the running scale $k$, and not at $k=0$ (which is the conventional value for IR FP's). Moreover, the aforementioned value of $\kappa_{c}$ is background dependent (in our case, $\Lambda$ dependent). Both these points are easy to understand. The issue of the finite value of $\kappa_{c}$ is related to nonanalyticity near the turning FP, especially to the square-root-like singularity of the flow as seen in the Eqs. (62) and (64). Actually, one can prove that if there is a turning point of the RG flow and the nonanalyticity is of the mentioned character, then this type of behavior near FP is only possible for finite nonzero $\kappa_{c}$. Conversely, if the FP happens at finite nonzero $\kappa_{c}$, then this is a turning point of the flow, and it must be continued analytically for $\kappa \leq \kappa_{c}$, if one looks for true deep IR FP. Then in such circumstances, the behavior near the turning FP can be characterized by any even orderroot-like nonanalyticity. Of course, the above found square root behavior is a paradigmatic example.
As for the background dependence, it should be stressed that only observable characteristics of the RG flow, such as a number of FP's or their type (scaling dimensions and related critical exponents, set of conformal primary operators, operator product expansion coefficients, etc., or all of this as called CFT data) should be background independent. On the other hand, the actual shape of the RG flow trajectories is, in general, background dependent (see, e.g., [49,95-100]).

(ii) It is evident from (56) that the IR FP obtained is entirely due to threshold phenomena, and the inclusion of the anomalous dimension $\eta$ (even with a supposedly exact nonperturbative expression for it) does not change the issue of the existence of this IR FP. Anomalous dimension is, however, responsible for the shape of the RG flow trajectory. Moreover, since threshold phenomena depend on a particular choice of the IR-cutoff kernel function $R_{k}(z)$, one might wonder how much such a choice influences the simultaneous running of $\beta_{C}$ and $\beta_{E}$ to FP values $\omega_{C * *}$ and $\omega_{E * *}$ in the IR. We accept the pragmatic assumption that usual IR-cutoff kernels influence the observable quantities only minimally, which is confirmed by almost all interesting examples.

(iii) The situation with IR FP in QWG may be compared to the decoupling of massive UV modes and the threshold phenomena, which occur for example in quantum electrodynamics (QED) due to finite-size mass of the electron - the lightest charged particle. It is well-known that in QED, the running of the effective electric charge $e(k)$ is stopped in the IR at an energy scale around mass of the electron $k=m_{e}$ and with some finite value attained $e_{\mathrm{cl}}$, which we call classical (long-distance) coupling of the electron to classical electromagnetic field. For higher energy values, the corresponding $\beta$ function for the electric charge is positive. Hence, we understand that in the QED case, the $\beta$ function of the running electric charge $e(k)$ tends to zero in the IR (and its corresponding coupling to its limiting IR value $e_{\mathrm{cl}}$ ), actually never crossing the zero, and always keeping the positive sign. In any mass-dependent scheme, one sees that the $\beta$ function in QED attains a zero value in the IR as the effect of integrating out all modes of charged particles. And roughly below the mass of the electron $m_{e}$, there are no active quantum degrees of freedom, and this is the reason why the running effectively is slowed down to a full halt at $k=0$. But it would be incorrect to say that since $e(k=0)=e_{\mathrm{cl}} \neq 0$, then in the IR, QED reaches a non-Gaussian FP. The stop of the RG running is due to exhaustion of all active modes and not due to some special structure of QFT of the Abelian $U(1)$ theory in the deep IR. The situation in QWG is very different. First, the $\beta$ functions on its way from UV 
to IR must inevitably cross zero. This moment in the RG flow we identified with the turning FP. After the turning point when we use analytic continuation, the flow is continued towards deep IR, where the IR FP is found. However, its existence is not a virtue of only inclusion of threshold phenomena. A reason why in QWG we found an interesting IR FP can be traced back to the form of the partition function on MSS background Eq. (24) and a consistent decoupling of heavy UV modes in any mass-dependent renormalization scheme. However, it is not true that we run out of all active degrees of freedom in QWG at low energies. As we know, in the spectrum, we have only massless modes. The FP in the IR we found for nontrivial values of the couplings and this is due to special structure of QWG. Hence, this IR FP (with $\omega_{C * *}$ and $\omega_{E * *}$ generally not being zero) can be rightly called non-Gaussian, as opposed to the one in QED.

(iv) Finally, one should remark that the existence of the IR FP is guaranteed for any value of the couplings $\omega_{E}$ and $\omega_{C}$. In other words, there are no initial values of $\omega_{E}$ and $\omega_{C}$ that would not run towards IR FP. This can be seen directly from the Eq. (56) which is only $k$ but not explicitly $\omega$ dependent. Therefore, we do not find any constraint from which it could be possible to find some special values of the couplings $\omega_{E}^{*}$ and $\omega_{C}^{*}$ only for which the IR FP would occur.

\section{SUMMARY AND DISCUSSION}

In this paper, we show that Weyl quantum gravity might provide a convenient theoretical setup for the UV-model building of phenomenologically viable quantum theory of gravity. The present paper, the first of a series, concentrates on the existence and description of the fixed point that is responsible for the spontaneous symmetry breakdown of the scale symmetry in the QWG. In particular, we proceed from the hypothesis that the QWG correctly describes a physics in the vicinity of some UV fixed point. This UV fixed point might correspond, for instance, to one of the critical points in a series of phase transitions that the Universe has undergone in the very early stage of its evolution. True UV completion could be then achieved within more fundamental theory, e.g,. Berkowits-Witten twistor-string theory or $\mathcal{N}=4$ conformal supergravity, which both harbor QWG in their low-energy limits (and do not have any pending unitarity issue). The idea that the early stage of the Universe should be conformally invariant has been recently promoted by R. Penrose [101,102], G. 't Hooft [103], and others [21,104,106].

In the next step, we have evolved the QWG from the presumed UV FP toward lower energies by using the functional renormalization group technique. A novel feature of our RG analysis is that ensuing effective action ansatz goes beyond the conventional one-loop truncation through inclusion of both the threshold phenomena and the effects of anomalous dimension. With these, the FRG flow equation was evaluated for two classes of Bach vacuum states, namely for the maximally symmetric spaces and Ricci-flat backgrounds. The IR fixed point was found to be non-Gaussian and IR stable in the space of considered couplings. One might view this IR FP as being akin to recently studied asymptotically safe FP found in the gaugematter-Yukawa system [105] (but this time not in the UV but in the IR sector) or as a kind of gravitational analogue of the Banks-Zaks FP known from Yang-Mills theories. Though the two operators $\sqrt{|g|} C^{2}$ and $\sqrt{|g|} E$ are at treelevel marginal, quantum corrections cause that both will become IR relevant with ensuing anomalous scaling $\gamma_{C}=\gamma_{E}=1 / 3$. In addition, the logical consistency of this scheme requires the incipient UV FP to be Gaussian.

The aforesaid IR FP can be identified with the critical point at which the Weyl invariance is spontaneously broken. A hallmark of the spontaneous scale symmetry breaking is the existence of the order-parameter field whose vacuum expectation value acquires a nonzero (dimensionful) value in the broken phase. For the case at hand, we have argued that the order-parameter field is a composite field of the Hubbard-Stratonovich type. As usual in SSB scenarios, a long-wavelength fluctuation of the latter should be identified in the broken phase with the Nambu-Goldstone mode (dilaton) $[107,108]$. For compatibility with an inflation-induced large structure formation, the Weyl symmetry should be broken before (or during) inflation. So, in particular, if the presumed UV fixed point is close to the inflationary scale $\left(\sim 10^{15}-10^{16} \mathrm{GeV}\right)$, then the asymptotic freedom in the vicinity of the UV FP would guarantee that our RG description of the IR FP in terms of enhanced one-loop truncation is well justified. We should also stress that our RG treatment of QWG with the preinflationary infrared fixed point fits in a broader theoretical framework of the (super)conformal inflation, which has been lately instrumental in classifying and generalizing classes of inflationary models favored by Planck data $[109,110]$.

The key observation in this context is that apart from the genuine IR FP (that is reached at zero value of the running scale $k$ ), the RG flow also exhibits bouncing behavior in the vicinity of the IR FP. In particular, both the $\beta$ functions for $C^{2}$ term and Gauss-Bonnet term ( $\beta$ function for the $R^{2}$ term is zero at our improved one-loop level) simultaneously reach the RG bounce fixed point at almost the same IR scale (up to $2 \%$ accuracy) irrespective of the background chosen. We noted that the observed squareroot type RG bouncing can be mapped on a multibranch (bouncing) holographic RG flow. Although we expect that the inclusion of higher loop effects or extension of our truncation ansatz will make the discrepancy between RG bounce FP's even smaller (in fact zero in an exact fully nonperturbative theory), we did not present an explicit 
multiloop computation confirming that this is the case, nor can we give a general proof.

There are still many questions to be understood. Here, is a partial list of them. Our treatment is essentially based on the FRG with a particular one-loop enhanced effective action and the Litim cutoff function (IR-cutoff kernel function $R_{k}$ ). Though the Litim cutoff is the most conventional cutoff used in the FRG computations, one might ask how much is the structure of the IR FP obtained (e.g., simultaneity of zeros of $\beta_{C}$ and $\beta_{E}$ and the values of $\omega_{C * *}$ and $\left.\omega_{E * *}\right)$ influenced by this particular choice. The conventional wisdom in the FRG posits that the structure and existence of FP's (but not the shape of the RG flow trajectories) should be independent of the particular choice of a cutoff function (provided it satisfies certain consistency conditions [49]). This expectation has been confirmed by a number of explicit computations in various systems $[45,111]$. On the other hand, any cutoff function represents an artificial term in the effective action, and every observable becomes in one way or another cutoff dependent after the unavoidable truncations and approximations in the FRG calculations. It might be thus interesting to make a comparison with other cutoffs on the market in order to see how robust is our prediction. To this end, one might use, for instance, two-parameter cutoff functions of Nagy and Nándori $[112,113]$ with parameters optimized via principle of minimal sensitivity [114], i.e., by requiring that the calculated observables depend least on the cutoff kernel parameters. Another option would be to use (conformally or also gauge-) invariant cutoff kernel functions based on the proper time regularization of divergent integrals as this was suggested in [115].

One can ask the question about the phenomenological implications of the considered here quantum Weyl gravity. This topic has been partially answered, and some applications to black hole physics (in particular to the issues of their formation and evaporation [116,117] and the origin of their finite entanglement entropy [118]) were found themselves to be successful. Another theoretical problem is the relation between quantum conformal theories and UV-finite theories. Some works in this direction were already discussed in $[81,82,119,120]$, and the ensuing benefits of solving the issue of GR singularities were shown in $[121,122]$. Moreover, we note the existing comprehensive review on the various problems of conformal symmetry in QFT and gravity in [123].

It also remains to be seen to what extent our RG treatment of the QWG for the considered class of backgrounds is impeded by the presumed nonunitarity of QWG. Note, that unitarity issue was not apparently essential for our reasonings, at least not for the considered backgrounds and given truncation ansatz. As already mentioned, the renormalizable QWG violates unitarity because it possesses a spin-two ghost on flat background. This might well be an artifact of our ill-devised expansion around a wrong vacuum state, namely the flat spacetime. In a sense, the situation could be reminiscent of that known from the symmetry breaking model with one real scalar field and a Higgs-like double-well potential $V(\Phi)=\lambda\left(\Phi^{2}-\mu^{2}\right)^{2}$, with $\mu^{2}, \lambda>0$ and with a tachyon in place of ghosts. We recall that the $S$-matrix unitarity means that the asymptotic "in" and "out" Fock spaces are unitarily equivalent. While the $S$ matrix is unitary for the scattering theories based upon "true" vacua $\Phi_{0}= \pm \mu$, this is certainly not the case when the tachyonic vacuum $\Phi_{0}=0$ is employed since incoming tachyonic Fock space states are not generally carried to the outgoing tachyonic Fock space [124]. So, an incorrectly chosen vacuum state alongside with an ensuing unstable tachyonic mode are "culprits" of nonunitarity. Could a similar disappearance of unstable fluctuations in nontrivial backgrounds be in operation also in the QWG?

\section{ACKNOWLEDGMENTS}

It is a pleasure to acknowledge helpful conversations with R. Percacci, P. Mannheim, I. Shapiro, K. Stelle, and M. Irakleidou. P. J. was supported by the Czech Science Foundation (GAČR), Grant No. 17-33812L. L. R. was supported from European Structural and Investment Fund (ESIF), EU Operational Programme Research, Development and Education No. CZ.02.2.69/0.0/0.0/16027/0008465. J. K. was supported by the Grant Agency of the Czech Technical University in Prague, Grant No. SGS19/183/OHK4/3T/14.
[1] N. Aghanim et al. (Planck Collaboration), arXiv:1807 .06209 .

[2] S. Coleman and E. Weinberg, Phys. Rev. D 7, 1888 (1973).

[3] S. L. Adler, Phys. Rev. Lett. 44, 1567 (1980).

[4] S. L. Adler, Rev. Mod. Phys. 54, 729 (1982).

[5] A. Zee, Ann. Phys. (N.Y.) 151, 431 (1983).

[6] B. L. Spokoiny, Phys. Lett. B 147, 39 (1984).
[7] H. Kleinert and H.-J. Schmidt, Gen. Relativ. Gravit. 34, 1295 (2002).

[8] S. Capozziello and V. Faraoni, Beyond Einstein Gravity; A Survey of Gravitational Theories for Cosmology and Astrophysics (Springer, London, 2011).

[9] M. Shaposhnikov and A. Shkerin, J. High Energy Phys. 10 (2018) 024. 
[10] P. Jizba, H. Kleinert, and F. Scardigli, Eur. Phys. J. C 75, 245 (2015).

[11] S. Y. Ayazi and A. Mohamadnejad, Eur. Phys. J. C 79, 140 (2019).

[12] K. Ishiwata, Phys. Lett. B 710, 134 (2012).

[13] P. D. Mannheim, Phys. Rev. D 85, 124008 (2012).

[14] P. D. Mannheim, Astrophys. J. 391, 429 (1992).

[15] J. G. O'Brien and P. D. Mannheim, Mon. Not. R. Astron. Soc. 421, 1273 (2012).

[16] E. S. Fradkin and A. A. Tseytlin, Phys. Rep. 119, 233 (1985).

[17] S. Elitzur, Phys. Rev. D 12, 3978 (1975).

[18] E. S. Fradkin and A. A. Tseytlin, Phys. Lett. B 134, 187 (1984).

[19] A. Schwimmer and S. Theisen, Nucl. Phys. B847, 590 (2011).

[20] M. Shaposhnikov and Ch. Wetterich, Phys. Lett. B 683, 196 (2010).

[21] K. A. Meissner and H. Nicolai, Phys. Lett. B 648, 312 (2007).

[22] K. S. Stelle, Phys. Rev. D 16, 953 (1977).

[23] H. van Dam and M. Veltman, Nucl. Phys. B22, 397 (1970); V. I. Zakharov, Pis'ma Zh. Eksp. Teor. Fiz. 12, 447 (1970) [JETP Lett. 12, 312 (1970)], http://www.jetpletters.ac.ru/ ps/1734/article_26353.shtml.

[24] D. G. Boulware and S. Deser, Phys. Rev. D 6, 3368 (1972).

[25] T. D. Lee and G. C. Wick, Nucl. Phys. B9, 209 (1969); Phys. Rev. D 2, 1033 (1970).

[26] D. Anselmi, J. High Energy Phys. 02 (2018) 141; Classical Quantum Gravity 36, 065010 (2019); J. High Energy Phys. 11 (2018) 021.

[27] M. B. Einhorn and D. R. T. Jones, Phys. Rev. D 96, 124025 (2017).

[28] V. I. Tkach, Mod. Phys. Lett. A 27, 1250131 (2012).

[29] E. Tomboulis, Phys. Lett. 70B, 361 (1977).

[30] M. Kaku, Phys. Rev. D 27, 2819 (1983).

[31] G. Cusin, F. de O. Salles, and I. L. Shapiro, Phys. Rev. D 93, 044039 (2016).

[32] A. V. Smilga, Nucl. Phys. B706, 598 (2005).

[33] A. V. Smilga, J. Phys. A 47, 052001 (2014).

[34] G. Narain, Eur. Phys. J. C 77, 683 (2017).

[35] L. Modesto and L. Rachwal, Int. J. Mod. Phys. D 26, 1730020 (2017).

[36] A. S. Koshelev, L. Modesto, L. Rachwal, and A. A. Starobinsky, J. High Energy Phys. 11 (2016) 067.

[37] F. Briscese and L. Modesto, Phys. Rev. D 99, 104043 (2019).

[38] M. Christodoulou and L. Modesto, JETP Lett. 109, 286 (2019).

[39] J. B. Hartle, Phys. Rev. D 49, 6543 (1994).

[40] H. D. Politzer, Phys. Rev. D 46, 4470 (1992).

[41] S. Lloyd, L. Maccone, R. Garcia-Patron, V. Giovannetti, and Y. Shikano, Phys. Rev. D 84, 025007 (2011).

[42] S. Weinberg, Ultraviolet divergences in quantum theories of gravitation, in General Relativity: An Einstein Centenary Survey, edited by S. W. Hawking and W. Israel (Cambridge University Press, Cambridge, England, 1979), Chap. 16, pp. 790-831.

[43] M. Reuter, Phys. Rev. D 57, 971 (1998); Nucl. Phys. B427, 291 (1994); Nucl. Phys. B417, 181 (1994).
[44] C. Wetterich, Phys. Lett. B 301, 90 (1993).

[45] A. Codello, R. Percacci, and C. Rahmede, Ann. Phys. (Amsterdam) 324, 414 (2009).

[46] D. Benedetti, P. F. Machado, and F. Saueressig, Nucl. Phys. B824, 168 (2010); S. Weinberg, arXiv:0903.0568.

[47] M. Niedermaier and M. Reuter, Living Rev. Relativity 9, 5 (2006).

[48] T. Banks and A. Zaks, Nucl. Phys. B196, 189 (1982).

[49] R. Percacci, An Introduction to Covariant Quantum Gravity and Asymptotic Safety (World Scientific, New York, 2017).

[50] A. Codello and R. Percacci, Phys. Rev. Lett. 97, 221301 (2006).

[51] J. F. Donoghue, arXiv:1911.02967.

[52] See Supplemental Material to this article at http://link.aps .org/supplemental/10.1103/PhysRevD.101.044050 for finer technical details, which include Refs. [16,49,53-63].

[53] D. F. Litim, Proc. Sci. (QG-Ph) (2007) 024.

[54] D. F. Litim, Phys. Rev. D 64, 105007 (2001); Phys. Lett. B 486, 92 (2000).

[55] D. Benedetti, P. F. Machado, and F. Saueressig, Mod. Phys. Lett. A 24, 2233 (2009).

[56] H. Kleinert, Path Integrals in Quantum Mechanics, Statistics, Polymer Physics, and Financial Markets (World Scientific, Singapore, 2006).

[57] H. Kleinert, Phys. Lett. A 114, 263 (1986); Mod. Phys. Lett. A 03, 531 (1988); Phys. Lett. B 189, 187 (1987).

[58] H. Kleinert, Phys. Lett. B 174, 335 (1986); Phys. Rev. Lett. 58, 1915 (1987).

[59] A. M. Polyakov, Phys. Lett. B 59, 79 (1975).

[60] H. Kleinert, Phys. Lett. B 196, 355 (1987).

[61] E. S. Fradkin and A. A. Tseytlin, Nucl. Phys. B234, 472 (1984).

[62] A. O. Barvinsky and G. A. Vilkovisky, Phys. Rep. 119, 1 (1985).

[63] A. Codello, R. Percacci, L. Rachwal, and A. Tonero, Eur. Phys. J. C 76, 226 (2016).

[64] H. Weyl, Math. Z. 2, 384 (1918).

[65] R. Bach, Math. Z. 9, 110 (1921).

[66] H. W. Hamber, Quantum Gravitation, The Feynman Path Integral Approach (Springer, Berlin, 2009).

[67] S. Carlip, Classical Quantum Gravity 15, 2629 (1998).

[68] M. H. Freedman, J. Diff. Geom. 17, 357 (1982).

[69] K. Hubbard, Phys. Rev. Lett. 3, 77 (1959).

[70] R. L. Stratonovich, Sov. Phys. Dokl. 2, 416 (1957).

[71] A. Altland and B. Simons, Condensed Matter Field Theory (Cambridge University Press, Cambridge, England, 2013).

[72] V. L. Ginzburg and L. D. Landau, Zh. Eksp. Teor. Fiz. 20, 1064 (1950).

[73] P. M. Stevenson, Phys. Rev. D 23, 2916 (1981).

[74] H. Kleinert and V. Schulte-Frohlinde, Critical Properties of $\phi^{4}$-Theories (World Scientific, Singapore, 2001).

[75] S. Fulling, L. Parker, and B. Hu, Phys. Rev. D 10, 3905 (1974).

[76] L. Modesto and L. Rachwal, J. High Energy Phys. 12 (2015) 173.

[77] M. Irakleidou and I. Lovrekovic, Phys. Rev. D 93, 104043 (2016).

[78] C. Wetterich, Phys. Rev. D 98, 026028 (2018).

[79] D. M. Capper and M. J. Duff, Phys. Lett. A 53, 361 (1975). 
[80] M. Asorey, E. V. Gorbar, and I. L. Shapiro, Classical Quantum Gravity 21, 163 (2003).

[81] L. Modesto and L. Rachwal, Nucl. Phys. B889, 228 (2014).

[82] L. Modesto and L. Rachwal, Nucl. Phys. B900, 147 (2015).

[83] L. Modesto, M. Piva, and L. Rachwal, Phys. Rev. D 94, 025021 (2016).

[84] L. Modesto and L. Rachwal, arXiv:1605.04173.

[85] G. de Berredo-Peixoto and I. L. Shapiro, Phys. Rev. D 70, 044024 (2004).

[86] E. S. Fradkin and A. A. Tseytlin, Phys. Lett. 104B, 377 (1981).

[87] E. S. Fradkin and A. A. Tseytlin, Nucl. Phys. B201, 469 (1982).

[88] E. Kiritsis, F. Nitti, and L. S. Pimenta, Fortschr. Phys. 65, 1600120 (2017).

[89] F. Nitti, L. S. Pimenta, and D. A. Steer, J. High Energy Phys. 07 (2018) 22.

[90] J. K. Ghosh, E. Kiritsis, F. Nitti, and L. T. Witkowski, J. High Energy Phys. 05 (2018) 34.

[91] R. Percacci and L. Rachwal, Fortschr. Phys. 62, 887 (2014).

[92] D. F. Litim, R. Percacci, and L. Rachwal, Phys. Lett. B 710, 472 (2012); J. Phys. Conf. Ser. 343, 012098 (2012).

[93] A. LeClair, J. M. Roman, and G. Sierra, Nucl. Phys. B700, 407 (2004).

[94] T. L. Curtright, X. Jin, and C. K. Zachos, Phys. Rev. Lett. 108, 131601 (2012).

[95] G. Narain and R. Percacci, Acta Phys. Pol. B 40, 3439 (2009), https://www.actaphys.uj.edu.pl/R/40/12/3439/pdf.

[96] N. Ohta, R. Percacci, and A. D. Pereira, Eur. Phys. J. C 77, 611 (2017).

[97] I. L. Shapiro and A. G. Zheksenaev, Phys. Lett. B 324, 286 (1994).

[98] G. de Berredo-Peixoto, A. Penna-Firme, and I. L. Shapiro, Mod. Phys. Lett. A 15, 2335 (2000).

[99] J. D. Goncalves, T. de Paula Netto, and I. L. Shapiro, Phys. Rev. D 97, 026015 (2018).

[100] V. F. Barra, P. M. Lavrov, E. A. Dos Reis, T. de Paula Netto, and I. L. Shapiro, arXiv:1910.06068.

[101] R. Penrose, Cycles of Time: An Extraordinary New View of the Universe (The Random House, London, 2010).

[102] R. Penrose, Found. Phys. 44, 557 (2014).
[103] G. 't Hooft, arXiv:1410.6675.

[104] R. Armillis, A. Monin, and M. Shaposhnikov, J. High Energy Phys. 10 (2013) 030.

[105] D. F. Litim and F. Sannino, J. High Energy Phys. 12 (2014) 178.

[106] G. Amelino-Camelia, M. Arzano, G. Gubitosi, and J. Magueijo, Int. J. Mod. Phys. D 24, 1543002 (2015).

[107] M. Blasone, P. Jizba, and G. Vitiello, Quantum Field Theory and its Macroscopic Manifestations (World Scientific \& ICP, London, 2010).

[108] M. Blasone, P. Jizba, and L. Smaldone, Phys. Rev. D 100, 045027 (2019).

[109] S. Ferrara, R. Kallosh, A. Linde, A. Marrani, and A. Van Proeyen, Phys. Rev. D 82, 045003 (2010); 83, 025008 (2011).

[110] I. Bars, P. Steinhardt, and N. Turok, Phys. Rev. D 89, 043515 (2014).

[111] M. Reuter and F. Saueressig, Quantum Gravity and the Functional Renormalization Group: The Road Towards Asymptotic Safety (Cambridge University Press, Cambridge, England, 2018).

[112] S. Nagy, B. Fazekas, L. Juhasz, and K. Sailer, Phys. Rev. D 88, 116010 (2013).

[113] I. Nándori, J. High Energy Phys. 04 (2013) 150.

[114] P. M. Stevenson, Phys. Rev. D 23, 2916 (1981).

[115] A. Bonanno and G. Lacagnina, Nucl. Phys. B693, 36 (2004); A. Bonanno, S. Lippoldt, R. Percacci, and G. P. Vacca, arXiv:1912.08135.

[116] C. Bambi, L. Modesto, S. Porey, and L. Rachwal, J. Cosmol. Astropart. Phys. 09 (2017) 033.

[117] C. Bambi, L. Modesto, S. Porey, and L. Rachwal, Eur. Phys. J. C 78, 116 (2018).

[118] S. Giaccari, L. Modesto, L. Rachwal, and Y. Zhu, Eur. Phys. J. C 78, 459 (2018).

[119] L. Modesto and L. Rachwal, arXiv:1605.04173.

[120] A. S. Koshelev, K. Sravan Kumar, L. Modesto, and L. Rachwal, Phys. Rev. D 98, 046007 (2018).

[121] L. Modesto and L. Rachwal, J. Phys. Conf. Ser. 942, 012015 (2017).

[122] C. Bambi, L. Modesto, and L. Rachwal, J. Cosmol. Astropart. Phys. 05 (2017) 003.

[123] L. Rachwal, Universe 4, 125 (2018).

[124] T. Jacobson, N. C. Tsamis, and R. P. Woodard, Phys. Rev. D 38, 1823 (1988). 